\title{
MULTILEVEL BALANCING DOMAIN DECOMPOSITION BY CONSTRAINTS DELUXE ALGORITHMS WITH ADAPTIVE COARSE SPACES FOR FLOW IN POROUS MEDIA*
}

\author{
STEFANO ZAMPINI ${ }^{\dagger}$ AND XUEMIN TU $\ddagger$
}

\begin{abstract}
Multilevel balancing domain decomposition by constraints (BDDC) deluxe algorithms are developed for the saddle point problems arising from mixed formulations of Darcy flow in porous media. In addition to the standard no-net-flux constraints on each face, adaptive primal constraints obtained from the solutions of local generalized eigenvalue problems are included to control the condition number. Special deluxe scaling and local generalized eigenvalue problems are designed in order to make sure that these additional primal variables lie in a benign subspace in which the preconditioned operator is positive definite. The current multilevel theory for BDDC methods for porous media flow is complemented with an efficient algorithm for the computation of the so-called malign part of the solution, which is needed to make sure the rest of the solution can be obtained using the conjugate gradient iterates lying in the benign subspace. We also propose a new technique, based on the Sherman-Morrison formula, that lets us preserve the complexity of the subdomain local solvers. Condition number estimates are provided under certain standard assumptions. Extensive numerical experiments confirm the theoretical estimates; additional numerical results prove the effectiveness of the method with higher order elements and high-contrast problems from real-world applications.
\end{abstract}

Key words. adaptive coarse space, BDDC, Darcy flow, domain decomposition, PETSc

AMS subject classifications. $65 \mathrm{~F} 08,65 \mathrm{~N} 55,65 \mathrm{Y} 05,68 \mathrm{~W} 10$

DOI. $10.1137 / 16 \mathrm{M} 1080653$

1. Introduction. The purpose of this work is to construct and analyze a balancing domain decomposition by constraints (BDDC) method [11] for the finite element (FE) discretization of saddle point problems arising from the mixed formulation of Darcy flows in porous media. Most previous work on domain decomposition methods for saddle point problems is based on the benign subspace approach [15, 38, 39, 40,42], where the original saddle point is reduced to a positive definite problem in the case of discontinuous pressure discretization spaces. Multilevel extensions of these ideas have been presented in $[48,47,57]$. For domain decomposition methods with continuous pressures, see $[29,58,59]$ and the references therein. We note that our two-level results are equally valid for the FE tearing and interconnecting dual-primal (FETI-DP) method [17], due to the well-known duality between BDDC and FETI-DP [34].

After the pioneering work [35], recent research on BDDC methods has focused on controlling the condition number of the preconditioned operators through the adaptive generation of coarse spaces $[6,8,23,24,25,37,44,48,64]$; these techniques lead to robust preconditioning techniques with tunable rates of convergence; see, in particular, [45]. The adaptive enrichment of the primal space is accomplished by means of solving

*Submitted to the journal's Methods and Algorithms for Scientific Computing section June 20, 2016; accepted for publication (in revised form) May 31, 2017; published electronically August 3, 2017.

http://www.siam.org/journals/sisc/39-4/M108065.html

Funding: The second author's work was supported in part by the National Science Foundation under contract DMS-1419069.

${ }^{\dagger}$ King Abdullah University of Science and Technology, Computer, Electrical and Mathematical Sciences and Engineering Division, Extreme Computing Research Center, Thuwal 23955, Saudi Arabia (stefano.zampini@kaust.edu.sa).

$¥$ Department of Mathematics, University of Kansas, Lawrence, KS 66045 (xtu@math.ku.edu). 
local generalized eigenvalue problems; the number of global synchronization steps and matrix vector multiplications in a Krylov solver can thus be accurately tuned at the expense of extra local flops and nearest neighbor communication during the setup of the preconditioner.

We base our algorithm on earlier works on BDDC methods for incompressible Stokes [30] and porous media flows [52], extending these algorithms to the modern, deluxe version of BDDC [61]. For the saddle point problem studied in this paper, modified local generalized eigenvalue problems, combined with deluxe scaling, are designed to make sure that the additional primal variables obtained are in the benign subspace, making it possible to use preconditioned conjugate gradient methods as the iterative solver. Condition number estimates are provided for the minimal as well as an adaptive coarse space.

We further extend the applicability of these methods to the case of highly heterogeneous coefficients and higher order div-conforming finite elements, with a specific focus on the algorithmic complexity of the solver and its multilevel extension [57]. Specifically, we present a new technique, which is based on the Sherman-Morrison formula, that allows us to preserve the complexity of the subdomain local solvers. We also complement the current multilevel theory for BDDC methods for porous media flow with an efficient algorithm for the computation of the so-called malign part of the solution, which is needed to guarantee that the rest of the solution can be obtained using the conjugate gradient iterates lying in the benign subspace.

Our work can be easily extended to almost incompressible elasticity and to incompressible Stokes flow with highly heterogeneous viscosity, even in the presence of stabilization terms in the discontinuous pressure spaces; cf. [13]. For a survey on BDDC methods, see $[63,65]$ and the references therein; for applications of the method to hybrid discretizations of porous media flows, see [46, 54].

The rest of the paper is organized as follows: section 2 introduces the variational formulation of the problem of interest and the associated discretization space. Section 3 describes the BDDC deluxe method for porous media flow, the benign subspace technique, and provides details on its efficient implementation given in the PETSc library [4]. An analysis of the method is carried out in section 4 and numerical results are provided in section 5 .

\section{Problem formulation.}

2.1. Model problem. The focus of this work is the solution of the following variational problem: find $(\boldsymbol{u}, p) \in \boldsymbol{U} \times P$ s.t. $\forall(\boldsymbol{v}, q) \in \boldsymbol{U} \times P$

$$
\left\{\begin{array}{l}
\mathbf{a}(\boldsymbol{u}, \boldsymbol{v})+\mathbf{b}(\boldsymbol{v}, p)=\int_{\Omega} \boldsymbol{g} \cdot \boldsymbol{v} d x+\int_{\Gamma_{D}} g_{\partial \Omega_{D}} \boldsymbol{v} \cdot \boldsymbol{n} d s \\
\mathbf{b}(\boldsymbol{u}, q)-\mathbf{c}(p, q)=\int_{\Omega} g q,
\end{array}\right.
$$

where $\Omega$ is a bounded Lipschitz domain in $\mathbb{R}^{3}$ and

$$
(2) \mathbf{a}(\boldsymbol{u}, \boldsymbol{v}):=\int_{\Omega} \boldsymbol{u} K_{p}(x)^{-1} \boldsymbol{v} d x, \mathbf{b}(\boldsymbol{u}, q):=\int_{\Omega} \nabla \cdot \boldsymbol{u} q d x, \mathbf{c}(p, q):=\int_{\Omega} w p q d x
$$

The boundary is split in $\partial \Omega:=\partial \Omega_{N} \cup \partial \Omega_{D}$, where $\partial \Omega_{N}$ is the part of the boundary with Neumann boundary conditions on the scalar variable $p$, while Dirichlet boundary conditions for $p$ are imposed on $\partial \Omega_{D}$. The functions $\boldsymbol{g}, g_{\partial \Omega_{D}}$, and $g$ represent suitable given data. $K_{p}(x)$ is a $3 \times 3$ symmetric, uniformly positive definite tensor, i.e., $0<$ $k_{m} \leq \boldsymbol{v}^{T} K_{p}(x) \boldsymbol{v} \leq k_{M}$ for any unit vector $\boldsymbol{v}$ and $w$ is a positive function in $L^{\infty}(\Omega)$. The spaces $\boldsymbol{U}$ and $P$ are defined as

$$
\boldsymbol{U}:=\left\{\boldsymbol{u} \in H(\operatorname{div}, \Omega): \boldsymbol{u} \cdot \boldsymbol{n}=0 \text { on } \partial \Omega_{N}\right\}, P:=L^{2}(\Omega),
$$


where $H(\operatorname{div}, \Omega)$ consists of vector-valued functions such that $\boldsymbol{u}$ and $\nabla \cdot \boldsymbol{u}$ belong to $\left(L^{2}(\Omega)\right)^{3}$ and $L^{2}(\Omega)$, respectively. Without loss of generality, we assume that the functions belonging to $\boldsymbol{U}$ have a vanishing normal component on $\partial \Omega_{N}$. We note that natural (Neumann) boundary conditions on $p$ give rise to essential boundary conditions on $\boldsymbol{u}$; cf. [7, Remark 1.3.3].

Problems of the form (1) arise from the mixed formulation of the Poisson problem (where $w=0$ ) and in the context of multiphase incompressible flow through porous media [7,9]; the functions belonging to $\boldsymbol{U}$ and $P$ are usually referred to as velocity and pressure. When $\partial \Omega_{D} \neq \emptyset$ or $w \neq 0$, the model problem (1) admits a unique solution; if $\partial \Omega_{D}=\emptyset$ and $w=0$, the pressure solution is determined only up to a constant, and the given data $g$ must satisfy the compatibility condition

$$
\int_{\Omega} g d x=0
$$

In the context of porous media flows, the function $w$ is zero in $\Omega$ except at the producer wells in the reservoirs (see, e.g., [28]). For existence and uniqueness of the solution for such a problem, together with a priori estimates, see [7, section 4.3].

2.2. FE spaces. In our analysis, we consider the div-conforming lowest order Raviart-Thomas (RT) elements on a triangulation $\mathcal{T}_{h}$ of tetrahedra. However, our results are equally valid for hexahedral elements [62]. The discrete velocity space is defined as

$$
\boldsymbol{U}_{h}:=\left\{\boldsymbol{u} \mid \boldsymbol{u}_{\mid \mathcal{K}} \in \mathcal{R} \mathcal{T}(\mathcal{K}), \mathcal{K} \in \mathcal{T}_{h} \text { and } \boldsymbol{u} \in H(\operatorname{div} ; \Omega)\right\},
$$

where $\mathcal{R} \mathcal{T}(\mathcal{K})$ is the lowest order RT space on the element $\mathcal{K}$. The degrees of freedom (dofs) are the average values of the normal components over the element faces

$$
\lambda_{f}(\boldsymbol{u}):=\frac{1}{|f|} \int_{f} \boldsymbol{u} \cdot \boldsymbol{n} d s, \quad f \subset \partial \mathcal{K} .
$$

A basis function of the lowest order RT element space is supported in two elements of $\mathcal{T}_{h}$. Piecewise constants are then used for the pressure space $P_{h}$. In what follows, we use the bold italic font $\boldsymbol{w}$ to denote $\mathrm{FE}$ functions, whereas the latin bold font $\mathbf{w}$ is used to denote a vector of dofs.

The discretization of (1) using $\boldsymbol{U}_{h} \times P_{h}$ leads to the linear system

$$
\left[\begin{array}{cc}
A & B^{T} \\
B & -C
\end{array}\right]\left[\begin{array}{l}
\mathbf{u}_{h} \\
\mathbf{p}_{h}
\end{array}\right]=\left[\begin{array}{l}
\mathbf{G}_{h} \\
\mathbf{g}_{h}
\end{array}\right]
$$

where $A, B$, and $C$ are the matrix representations of $\mathbf{a}(\cdot, \cdot), \mathbf{b}(\cdot, \cdot)$, and $\mathbf{c}(\cdot, \cdot)$ given in (2). The linear system (4) is symmetric indefinite with $A$ symmetric positive definite, and it is singular when $C=0$ and $\partial \Omega_{D}=\emptyset$; in this case, the kernel is spanned by the constant pressure function.

In our numerical experiments, we also consider RT elements of higher order, as well as Brezzi-Douglas-Marini (BDM) elements on tetrahedra; these elements have additional dofs corresponding to moments of the normal component over element faces and in the interior. When using higher order velocity spaces, the polynomial order for the pressure space should be increased accordingly; inf-sup stable pairs for (1) are $\mathrm{RT}_{\mathrm{k}} \times \mathrm{P}_{k}$ or $\mathrm{BDM}_{\mathrm{k}+1} \times \mathrm{P}_{k} ;$ cf. [7].

Since our method relies on purely algebraic arguments, its application is well suited for div-conforming spaces arising from upscaling techniques that preserve the 
de Rham complex through a hierarchy of coarse spaces [28]. The range of applicability of the method also extends to quadrilateral and hexahedral elements designed for highly distorted grids, for which the classical $\mathrm{RT}_{0}$ and $\mathrm{BDM}_{1}$ spaces fail to obtain optimal $L^{2}$ convergence for the divergence of the velocity; see, e.g., [3, 16]. We also remark that our BDDC methods can be applied to recently developed discretization techniques for distorted quadrilaterals and hexahedra such as multipoint flux mixed FE methods [21, 60].

\section{Balancing domain decomposition methods.}

3.1. Space decompositions. Working in the framework of iterative substructuring as in [51, Chapters 4-6], we decompose $\Omega$ into $N$ nonoverlapping open Lipschitz subdomains $\Omega_{i}$, i.e.,

$$
\bar{\Omega}=\bigcup_{i=1}^{N} \bar{\Omega}_{i}, \quad \Gamma:=\bigcup_{i \neq j} \partial \Omega_{j} \cap \partial \Omega_{i},
$$

where $\Gamma$ is the interface between the subdomains. We further assume that each $\Omega_{i}$ is connected; we note that such a requirement is not a limitation, as each disconnected component of a subdomain can be thought as a separate subdomain.

Matrix assembly is performed separately on each subdomain, obtaining

$$
\left[\begin{array}{ll}
A^{(i)} & B^{(i) T} \\
B^{(i)} & -C^{(i)}
\end{array}\right]
$$

where $A^{(i)}, B^{(i)}$, and $C^{(i)}$ are the matrix representations of $\mathbf{a}_{i}(\cdot, \cdot), \mathbf{b}_{i}(\cdot, \cdot)$, and $\mathbf{c}_{i}(\cdot, \cdot)$, defined as in (2), by replacing $\Omega$ with $\Omega_{i}$ as the integration set. The subdomain matrices (5) are symmetric indefinite, with $A^{(i)}$ symmetric positive definite; when $w=0$ in the subdomain, the $C^{(i)}$ block vanishes.

DEFINITION 3.1 (benign/malign subdomains). We say that a subdomain $\Omega_{i}$ is malign if and only if $C^{(i)}=0$ and $\partial \Omega_{i} \cap \partial \Omega_{D}=\emptyset$. Otherwise, it is benign.

The discretization spaces are decomposed as (cf. [19, 30, 52])

$$
\boldsymbol{U}_{h}=\boldsymbol{W}_{I} \oplus \widehat{\boldsymbol{W}}_{\Gamma}, \quad P_{h}=Q_{I} \oplus Q_{0} .
$$

$\widehat{\boldsymbol{W}}_{\Gamma}$ is the space of traces on $\Gamma$ of functions in $\boldsymbol{U}_{h}$, whereas $\boldsymbol{W}_{I}$ is the product space of subdomain interior velocity spaces $\boldsymbol{W}_{I}^{(i)}$. The FE pressure spaces corresponding to malign subdomains are split as

$$
P_{h}\left(\Omega_{i}\right)=Q_{I}^{(i)} \oplus Q_{0}^{(i)}, \quad Q_{I}^{(i)}=\left\{q \in P_{h}\left(\Omega_{i}\right): \int_{\Omega_{i}} q d x=0\right\},
$$

where $Q_{0}^{(i)}$ is spanned by the constant pressure function on the subdomain. For benign subdomains, $Q_{I}^{(i)}=P_{h}\left(\Omega_{i}\right)$ and $Q_{0}^{(i)}=\emptyset$. The spaces $Q_{I}$ and $Q_{0}$ are then obtained as the product spaces of $Q_{I}^{(i)}$ and $Q_{0}^{(i)}$, respectively.

Using a change of basis in the pressure space, the matrix (5) for a malign subdomain can be written as

$$
\left[\begin{array}{cccc}
A_{I I}^{(i)} & B_{I I}^{(i) T} & A_{I \Gamma}^{(i)} & 0 \\
B_{I I}^{(i)} & 0 & B_{I \Gamma}^{(i)} & 0 \\
A_{I \Gamma}^{(i) T} & B_{I \Gamma}^{(i) T} & A_{\Gamma \Gamma}^{(i)} & B_{0 \Gamma}^{(i) T} \\
0 & 0 & B_{0 \Gamma}^{(i)} & 0
\end{array}\right]
$$

Copyright (c) by SIAM. Unauthorized reproduction of this article is prohibited. 
The lower left and upper right blocks of (7) vanish since, by the divergence theorem, $\mathbf{b}\left(\boldsymbol{u}_{I}^{(i)}, p_{0}\right)=0$ for $p_{0} \in Q_{0}^{(i)}$ and $\boldsymbol{u}_{I}^{(i)} \in \boldsymbol{W}_{I}^{(i)}$. A pressure change of basis is not needed for benign subdomains. We note that the pressure change of basis comes with a loss of sparsity and it is thus to be avoided in practical implementations; see section 3.6.1 for more details.

The linear system (4) is now rewritten as

$$
\left[\begin{array}{cccc}
A_{I I} & B_{I I}^{T} & A_{I \Gamma} & 0 \\
B_{I I} & -C_{I I} & B_{I \Gamma} & 0 \\
A_{I \Gamma}^{T} & B_{I \Gamma}^{T} & A_{\Gamma \Gamma} & B_{0 \Gamma}^{T} \\
0 & 0 & B_{0 \Gamma} & 0
\end{array}\right]\left[\begin{array}{c}
\mathbf{u}_{h, I} \\
\mathbf{p}_{h, I} \\
\mathbf{u}_{h, \Gamma} \\
\mathbf{p}_{h, 0}
\end{array}\right]=\left[\begin{array}{c}
\mathbf{G}_{h, I} \\
\mathbf{g}_{h, I} \\
\mathbf{G}_{h, \Gamma} \\
\mathbf{g}_{h, 0}
\end{array}\right]
$$

where $B_{0 \Gamma}$ is obtained by assembling the $B_{0 \Gamma}^{(i)}$ matrices on $\widehat{\boldsymbol{W}}_{\Gamma}$, and where the number of rows of $B_{0 \Gamma}$ equals the number of malign subdomains. $C_{I I}$ is a block-diagonal matrix with blocks given by $C_{I I}^{(i)}$; we note that the latter vanish for malign subdomains.

3.2. Schur complement reduction. We define the local Schur complement matrices $S_{\Gamma}^{(i)}$ by statically condensing the interior dofs corresponding to the FE functions belonging to $\boldsymbol{W}_{I}^{(i)} \times Q_{I}^{(i)}$, obtaining

$$
\left[\begin{array}{ccc}
A_{I I}^{(i)} & B_{I I}^{(i) T} & A_{I \Gamma}^{(i)} \\
B_{I I}^{(i)} & -C_{I I}^{(i)} & B_{I \Gamma}^{(i)} \\
A_{I \Gamma}^{(i) T} & B_{I \Gamma}^{(i) T} & A_{\Gamma \Gamma}^{(i)}
\end{array}\right]\left[\begin{array}{c}
\mathbf{w}_{I}^{(i)} \\
\mathbf{p}_{I}^{(i)} \\
\mathbf{w}_{\Gamma}^{(i)}
\end{array}\right]=\left[\begin{array}{c}
0 \\
0 \\
S_{\Gamma}^{(i)} \mathbf{w}_{\Gamma}^{(i)}
\end{array}\right]
$$

For a malign subdomain, (7) is recast as the saddle point matrix

$$
\left[\begin{array}{cc}
S_{\Gamma}^{(i)} & B_{0 \Gamma}^{(i) T} \\
B_{0 \Gamma}^{(i)} & 0
\end{array}\right]
$$

The definition of $S_{\Gamma}^{(i)}$ is well determined, since the interior problems are always solvable; we note that for malign subdomains this is guaranteed by having removed the constant pressure. Moreover, since $A^{(i)}$ and $C^{(i)}$ are symmetric positive definite matrices, by Sylvester's law of inertia, $S_{\Gamma}^{(i)}$ is symmetric positive definite.

We now consider the following block factorization for the inverse of the matrix given in (8), denoted by $K$,

$$
\left[\begin{array}{cc}
I & -K_{I I}^{-1} K_{I \Gamma} \\
0 & I
\end{array}\right]\left[\begin{array}{cc}
K_{I I}^{-1} & 0 \\
0 & \widehat{S}^{-1}
\end{array}\right]\left[\begin{array}{cc}
I & 0 \\
-K_{I \Gamma}^{T} K_{I I}^{-1} & I
\end{array}\right], \quad K_{I \Gamma}:=\left[\begin{array}{cc}
A_{I \Gamma} & 0 \\
B_{I \Gamma} & 0
\end{array}\right]
$$

where

$$
\widehat{S}:=\left[\begin{array}{cc}
\widehat{S}_{\Gamma} & B_{0 \Gamma}^{T} \\
B_{0 \Gamma} & 0
\end{array}\right]
$$

with $\widehat{S}_{\Gamma}:=R_{\Gamma}^{T} S_{\Gamma} R_{\Gamma}$, where $S_{\Gamma}$ is the direct sum of subdomain Schur complements. $R_{\Gamma}$ is the direct sum of the operators $R_{\Gamma}^{(i)}$ that restrict from $\widehat{\boldsymbol{W}}_{\Gamma}$ to $\boldsymbol{W}_{\Gamma}^{(i)} . K_{I I}$ is a block-diagonal matrix, with subdomain blocks

$$
K_{I I}^{(i)}:=\left[\begin{array}{cc}
A_{I I}^{(i)} & B_{I I}^{(i) T} \\
B_{I I}^{(i)} & -C_{I I}^{(i)}
\end{array}\right]
$$


The preconditioning strategy of iterative substructuring methods consists in using direct solvers for $K_{I I}^{-1}$, and by replacing $\widehat{S}^{-1}$ with the action of a preconditioner, i.e.,

$$
M^{-1}:=\left[\begin{array}{cc}
I & -K_{I I}^{-1} K_{I \Gamma} \\
0 & I
\end{array}\right]\left[\begin{array}{cc}
K_{I I}^{-1} & 0 \\
0 & M_{\Gamma}^{-1}
\end{array}\right]\left[\begin{array}{cc}
I & 0 \\
-K_{I \Gamma}^{T} K_{I I}^{-1} & I
\end{array}\right] .
$$

As shown in [12], the preconditioned operator $M^{-1} K$ has the same eigenvalues as $M_{\Gamma}^{-1} \widehat{S}$. By properly choosing the initial guess (see [51, section 2.5.2]), the action of $K_{I I}^{-1}$ is needed just once per preconditioner application.

\subsection{BDDC methods.}

3.3.1. Primal and dual spaces. We consider the partially assembled velocity space $\widetilde{\mathbf{W}}_{\Gamma}$,

$$
\widehat{\boldsymbol{W}}_{\Gamma} \subset \widetilde{\boldsymbol{W}}_{\Gamma} \subset \boldsymbol{W}_{\Gamma}, \widetilde{\boldsymbol{W}}_{\Gamma}=\boldsymbol{W}_{\Delta} \oplus \widehat{\boldsymbol{W}}_{\Pi},
$$

where $\widehat{\boldsymbol{W}}_{\Pi}$ is the primal space and $\boldsymbol{W}_{\Delta}$ is the product space of the local dual spaces $\boldsymbol{W}_{\Delta}^{(i)}$, consisting of functions with zero values at the primal dofs. Functions belonging to $\widetilde{\boldsymbol{W}}_{\Gamma}$ are continuous at the primal level and discontinuous elsewhere on $\Gamma$. In order to describe the algorithm, we need to introduce some additional operators. $\widetilde{R}_{\Gamma}^{(i)}$ maps $\widetilde{\boldsymbol{W}}_{\Gamma}$ to $\boldsymbol{W}_{\Delta}^{(i)} \oplus \boldsymbol{W}_{\Pi}^{(i)}$, whereas $\widetilde{R}_{\Delta}^{(i)}$ maps $\widetilde{\boldsymbol{W}}_{\Gamma}$ to $\boldsymbol{W}_{\Delta}^{(i)}$. The restriction of $\widehat{\boldsymbol{W}}_{\Pi}$ to $\boldsymbol{W}_{\Pi}^{(i)}$ is denoted by $R_{\Pi}^{(i)} ; \widetilde{R}_{\Pi}$ maps $\widetilde{\boldsymbol{W}}_{\Gamma}$ to $\widehat{\boldsymbol{W}}_{\Pi}$, whereas $\bar{R}_{\Gamma}$ maps $\widehat{\boldsymbol{W}}_{\Gamma}$ to $\widetilde{\boldsymbol{W}}_{\Gamma}$.

Equivalence classes of $\boldsymbol{W}_{\Gamma}$ play a very important role in the design, analysis, and parallel implementation of the BDDC methods. In this work, each interface dof is associated with an element face common to only two elements; equivalence classes are thus given as sets of dofs at the intersection of the boundaries of two neighboring subdomains. We refer to such an interface class as a subdomain face.

Following [52], we require that the dual space satisfy the no-net-flux condition

$$
\int_{\partial \Omega_{i}} \boldsymbol{w}_{\Delta}^{(i)} \cdot \boldsymbol{n}=0 \forall \boldsymbol{w}_{\Delta}^{(i)} \in \boldsymbol{W}_{\Delta}^{(i)} .
$$

Assuming that each subdomain face $F$ is a connected subset of $\partial \Omega_{i}$, we require that

$$
\int_{F} \boldsymbol{w}_{\Delta}^{(i)} \cdot \boldsymbol{n}=0 \quad \forall F \subset \Gamma_{i} .
$$

Remark 3.2. By the divergence theorem, $\int_{\Omega_{i}} \nabla \cdot \boldsymbol{u} d x=\sum_{F} \int_{F} \boldsymbol{u} \cdot \boldsymbol{n} d s$; the quadrature weights needed to apply the face constraints (15) can thus be extracted from the vector $B^{(i) T} \mathbf{1}$, where $\mathbf{1}$ is a constant unit vector of appropriate size.

In section 4.1.2, we consider adaptively generated enrichments of this minimal velocity primal space to control the condition number of the preconditioned BDDC operator.

Some care must be exercised when working with decompositions obtained by mesh partitioners, since the detection of disconnected subdomains is crucial for the success of the algorithm; since each dof is associated with an element face or with the element interior, disconnected local partitions can easily be detected by analyzing the connected components of the connectivity graph associated with the sparsity pattern of the subdomain matrices. Connected subdomain faces are then detected using the connectivity graph of the interface dofs, where two dofs are considered connected if they belong to the same (connected) subdomain, and their element faces have an element edge in common. 
To simplify the description of the algorithm, we assume that a change of basis for the velocity space has been performed, and that the primal dofs, associated with the average normal flux over each face, have been made explicit. We note that the change of basis induces a loss of sparsity and it is not used in practice; see section 3.6.2 for more details. We can reorder the local subdomain matrices, i.e., for malign subdomains

$$
\left[\begin{array}{ccccc}
A_{I I}^{(i)} & B_{I I}^{(i) T} & A_{I \Delta}^{(i)} & A_{I \Pi}^{(i)} & 0 \\
B_{I I}^{(i)} & 0 & B_{I \Delta}^{(i)} & B_{I \Pi}^{(i)} & 0 \\
A_{I \Delta}^{(i) T} & B_{I \Delta}^{(i) T} & A_{\Delta \Delta}^{(i)} & A_{\Delta \Pi}^{(i)} & 0 \\
A_{I \Pi}^{(i) T} & B_{I \Pi}^{(i) T} & A_{\Delta \Pi}^{(i) T} & A_{\Pi \Pi}^{(i)} & B_{0 \Pi}^{(i) T} \\
0 & 0 & 0 & B_{0 \Pi}^{(i)} & 0
\end{array}\right],
$$

and, similarly, for any benign subdomain. Since the functions in the dual space satisfy (14), by the divergence theorem the blocks of (16) that corresponds to the coupling between $Q_{0}^{(i)}$ and $\boldsymbol{W}_{\Delta}^{(i)}$ vanish.

3.3.2. Partial assembly. We introduce the partially assembled Schur complement of the matrix in (8),

$$
\widetilde{S}:=\left[\begin{array}{cc}
\widetilde{S}_{\Gamma} & \widetilde{B}_{0 \Gamma}^{T} \\
\widetilde{B}_{0 \Gamma} & 0
\end{array}\right],
$$

where $\widetilde{B}_{0 \Gamma}$ is obtained by assembling the malign subdomain operators $B_{0 \Gamma}^{(i)}$ on $\widetilde{\boldsymbol{W}}_{\Gamma}$. The action of $\widetilde{S}_{\Gamma}$ is defined by

$$
\left[\begin{array}{cccccc}
K_{I I}^{(1)} & K_{I \Delta}^{(1)} & & & & \widetilde{K}_{I \Pi}^{(1)} \\
K_{I \Delta}^{(1)^{T}} & K_{\Delta \Delta}^{(1)} & & & & \widetilde{K}_{\Delta \Pi}^{(1)} \\
& & \ddots & & & \vdots \\
& & & K_{I I}^{(N)} & K_{I \Delta}^{(N)} & \widetilde{K}_{I \Pi}^{(N)} \\
& & & K_{I \Delta}^{(N)^{T}} & K_{\Delta \Delta}^{(N)} & \widetilde{K}_{\Delta \Pi}^{(N)} \\
\widetilde{K}_{I \Pi}^{(1)^{T}} & \widetilde{K}_{\Delta \Pi}^{(1)^{T}} & \ldots & \widetilde{K}_{I \Pi}^{(N)^{T}} & \widetilde{K}_{\Delta \Pi}^{(N)^{T}} & \widetilde{K}_{\Pi \Pi}
\end{array}\right]\left[\begin{array}{c}
\mathbf{w}_{I}^{(1)} \\
\mathbf{w}_{\Delta}^{(1)} \\
\vdots \\
\mathbf{w}_{I}^{(N)} \\
\mathbf{w}_{\Delta}^{(N)} \\
\mathbf{w}_{\Pi}
\end{array}\right]=\left[\begin{array}{c}
0 \\
\widetilde{R}_{\Delta}^{(1)} \widetilde{S}_{\Gamma} \mathbf{w}_{\Gamma} \\
\vdots \\
0 \\
\widetilde{R}_{\Delta}^{(N)} \widetilde{S}_{\Gamma} \mathbf{w}_{\Gamma} \\
\widetilde{R}_{\Pi \Pi} \widetilde{S}_{\Gamma} \mathbf{w}_{\Gamma}
\end{array}\right]
$$

where $K_{\Delta \Delta}^{(i)}:=A_{\Delta \Delta}^{(i)}, K_{I \Delta}^{(i) T}:=\left[A_{I \Delta}^{(i) T} B_{I \Delta}^{(i) T}\right], \widetilde{K}_{I \Pi}^{(i)}:=K_{I \Pi}^{(i)} R_{\Pi}^{(i)}, K_{I \Pi}^{(i) T}:=\left[A_{I \Pi}^{(i) T} B_{I \Pi}^{(i) T}\right]$, $\widetilde{K}_{\Delta \Pi}^{(i)}:=K_{\Delta \Pi}^{(i)} R_{\Pi}^{(i)}, K_{\Delta \Pi}^{(i)}:=A_{\Delta \Pi}^{(i)}$, and $\widetilde{K}_{\Pi \Pi}:=\sum_{i=1}^{N} R_{\Pi}^{(i)}{ }^{T} A_{\Pi \Pi}^{(i)} R_{\Pi}^{(i)}$.

3.3.3. Scaling operator. Central for the success of the algorithm is the scaling operator $\widetilde{R}_{D, \Gamma}$, mapping $\widehat{W}_{\Gamma}$ into $\widetilde{W}_{\Gamma}$, and defined as the direct sum of the operators $D^{(i)} \widetilde{R}_{\Gamma}^{(i)} \bar{R}_{\Gamma}$; the matrices $D^{(i)}$ should provide a partition of unity, i.e.,

$$
\widetilde{R}_{D, \Gamma}^{T} \widetilde{R}_{\Gamma}=\widetilde{R}_{\Gamma}^{T} \widetilde{R}_{D, \Gamma}=I
$$

where $\widetilde{R}_{\Gamma}$ is the direct sum of the $\widetilde{R}_{\Gamma}^{(i)}$ operators. A description of conventional scaling techniques, where the $D^{(i)}$ are diagonal matrices, can be found in [11,34]; for their application in the context of porous media flows, see [52].

In this work, we consider a modification of the deluxe scaling, which has been successfully applied to vector field problems in $H$ (curl) [14], $H$ (div) [41], as well as in 
the context of isogeometric analysis [5]. In BDDC deluxe algorithms, we work with the principal minors of $S_{\Gamma}^{(i)}$ associated with each individual subdomain face $F$, i.e.,

$$
S_{F F}^{(i)}:=R_{F}^{(i)} S_{\Gamma}^{(i)} R_{F}^{(i) T},
$$

where $R_{F}^{(i)}$ maps $\boldsymbol{W}_{\Gamma}^{(i)}$ to the dofs located on $F$. We split these matrices as follows:

$$
S_{F F}^{(i)}=\left[\begin{array}{ll}
S_{\Pi_{F}^{c} \Pi_{F}^{c}} & S_{\Pi_{F}^{c} \Pi_{F}} \\
S_{\Pi_{F}^{c} \Pi_{F}}^{T} & S_{\Pi_{F} \Pi_{F}}
\end{array}\right]
$$

where $\Pi_{F}$ is the primal dof associated with the average normal flux over $F$, and $\Pi_{F}^{c}=F \backslash \Pi_{F}$. The deluxe scaling matrices $D^{(i)}$ are block-diagonal, with a block $D_{F}^{(i)}$ for each subdomain face $F$, shared by $\Omega_{i}$ and $\Omega_{j}$, and defined in our case as

$$
D_{F}^{(i)}:=\left[\begin{array}{cc}
S_{\Pi_{F}^{c} \Pi_{F}^{c}}^{(i)}\left(S_{\Pi_{F}^{c} \Pi_{F}^{c}}^{(i)}+S_{\Pi_{F}^{c} \Pi_{F}^{c}}^{(j)}\right)^{-1} & 0 \\
0 & S_{\Pi_{F} \Pi_{F}}^{(i)}\left(S_{\Pi_{F} \Pi_{F}}^{(i)}+S_{\Pi_{F} \Pi_{F}}^{(j)}\right)^{-1}
\end{array}\right]
$$

The deluxe scaling, defined in (20), is a standard deluxe scaling on the orthogonal complement of the normal fluxes for each face. For the details of our implementation, see section 3.6.2. We also point out that the lower right block could be replaced by the values which serve as a partition of unity, i.e., $1 / 2$.

3.3.4. BDDC preconditioner. The BDDC preconditioner for $\widehat{S}$ in (11) is

$$
M_{\Gamma}^{-1}:=\widetilde{R}_{D}^{T} \widetilde{S}^{-1} \widetilde{R}_{D}, \quad \widetilde{R}_{D}=\left[\begin{array}{cc}
\widetilde{R}_{D, \Gamma} & 0 \\
0 & I
\end{array}\right] .
$$

Given the definition of $\widetilde{S}_{\Gamma}$ in (18), by block elimination [30,31], we have

$$
\widetilde{S}^{-1}=\sum_{i=1}^{N}\left[\begin{array}{ll}
0 & R_{\Delta 0}^{(i) T}
\end{array}\right]\left[\begin{array}{cc}
K_{I I}^{(i)} & K_{I \Delta}^{(i)} \\
K_{I \Delta}^{(i) T} & K_{\Delta \Delta}^{(i)}
\end{array}\right]^{-1}\left[\begin{array}{c}
0 \\
R_{\Delta 0}^{(i)}
\end{array}\right]+\Phi S_{C C}^{-1} \Phi^{T},
$$

where $R_{\Delta 0}^{(i)}$ restricts $\widetilde{\boldsymbol{W}}_{\Gamma} \times Q_{0}$ to $\boldsymbol{W}_{\Delta}^{(i)}$. The primal basis functions are given by

$$
\Phi:=R_{\Pi 0}^{T}-\sum_{i=1}^{N}\left[\begin{array}{ll}
0 & R_{\Delta 0}^{(i) T}
\end{array}\right]\left[\begin{array}{cc}
K_{I I}^{(i)} & K_{I \Delta}^{(i)} \\
K_{I \Delta}^{(i) T} & K_{\Delta \Delta}^{(i)}
\end{array}\right]^{-1}\left[\begin{array}{c}
K_{I \Pi}^{(i)} \\
K_{\Delta \Pi}^{(i)}
\end{array}\right],
$$

where $R_{\Pi \text { п }}$ maps $\widetilde{\boldsymbol{W}}_{\Gamma} \times Q_{0}$ into $\widehat{\boldsymbol{W}}_{\Pi} \times Q_{0}$. The coarse problem $S_{C C}$ is assembled from subdomain contributions as

$$
S_{C C}:=\left[\begin{array}{cc}
S_{\Pi \Pi} & B_{0 \Pi}^{T} \\
B_{0 \Pi} & 0
\end{array}\right]=\sum_{i=1}^{N} R_{C}^{(i)^{T}} S_{C C}^{(i)} R_{C}^{(i)}, \quad S_{\Pi \Pi}:=\sum_{i=1}^{N} R_{\Pi}^{(i)^{T}} S_{\Pi \Pi}^{(i)} R_{\Pi}^{(i)},
$$

where $R_{C}^{(i)}$ maps from $\widehat{\boldsymbol{W}}_{\Pi} \times Q_{0}$ to $\boldsymbol{W}_{\Pi}^{(i)} \times Q_{0}^{(i)}$ and $B_{0 \Pi}$ is obtained by assembling the malign subdomain operators $B_{0 \Pi}^{(i)}$ on $\widehat{\boldsymbol{W}}_{\Pi}$. Using the notation $K_{\Pi \Pi}^{(i)}:=A_{\Pi \Pi}^{(i)}$, the coarse subdomain matrices $S_{C C}^{(i)}$ for a benign subdomain are given by

$$
S_{C C}^{(i)}=S_{\Pi \Pi}^{(i)}, \quad S_{\Pi \Pi}^{(i)}:=K_{\Pi \Pi}^{(i)}-\left[\begin{array}{ll}
K_{I \Pi}^{(i) T} & K_{\Delta \Pi}^{(i) T}
\end{array}\right]\left[\begin{array}{cc}
K_{I I}^{(i)} & K_{I \Delta}^{(i)} \\
K_{I \Delta}^{(i) T} & K_{\Delta \Delta}^{(i)}
\end{array}\right]^{-1}\left[\begin{array}{c}
K_{I \Pi}^{(i)} \\
K_{\Delta \Pi}^{(i)}
\end{array}\right],
$$


while a malign subdomain contributes the saddle point matrix

$$
S_{C C}^{(i)}=\left[\begin{array}{cc}
S_{\Pi \Pi}^{(i)} & B_{0 \Pi}^{(i) T} \\
B_{0 \Pi}^{(i)} & 0
\end{array}\right],
$$

having one extra row and column corresponding to the coupling between $Q_{0}^{(i)}$ and $\boldsymbol{W}_{\Pi}^{(i)}$; the nonzero entries of $B_{0 \Pi}^{(i)}$ are located in the columns associated with the primal dofs representing the average normal flux over subdomain faces.

Remark 3.3. $S_{C C}$ is singular if all subdomains are malign: the null space is spanned by the functions that are constant on $Q_{0}$ and vanish on $\widehat{\boldsymbol{W}}_{\Pi}$. In this case, the right-hand side of (8) has to satisfy the compatibility condition (3); thus, the righthand side of the coarse linear system is always compatible throughout the Krylov process.

3.4. Benign subspaces. The matrix $\widehat{S}$ given in (11) is symmetric indefinite on the space $\widehat{\boldsymbol{W}}_{\Gamma} \times Q_{0}$, but it is positive definite on $\left\{\widehat{\boldsymbol{W}}_{\Gamma} \cap \operatorname{ker}\left(B_{0 \Gamma}\right)\right\} \times Q_{0}$. As shown in $[30,52]$, we can construct a BDDC preconditioner which is symmetric positive definite on the latter space; moreover, given a suitable starting vector, the conjugate gradient iterates remain in the same space when solving (4).

Definition 3.4 (benign subspaces). We call

$$
\widehat{\boldsymbol{W}}_{\Gamma, B}:=\left\{\boldsymbol{w}_{\Gamma} \in \widehat{\boldsymbol{W}}_{\Gamma} \mid B_{0 \Gamma} \mathbf{w}_{\Gamma}=0\right\}, \quad \widetilde{\boldsymbol{W}}_{\Gamma, B}:=\left\{\boldsymbol{w}_{\Gamma} \in \widetilde{\boldsymbol{W}}_{\Gamma} \mid \widetilde{B}_{0 \Gamma} \mathbf{w}_{\Gamma}=0\right\},
$$

the benign subspaces of $\widehat{\boldsymbol{W}}_{\Gamma}$ and $\widetilde{\boldsymbol{W}}_{\Gamma}$, respectively.

The following lemma is crucial for proving the positive definitess of the preconditioned BDDC operator.

LEMma 3.5. Let the dual space satisfy the no-net-flux condition (14) and $\boldsymbol{w}_{\Gamma} \in$ $\widetilde{\boldsymbol{W}}_{\Gamma, B}$. Then, $\widetilde{\boldsymbol{R}}_{D, \Gamma}^{T} \boldsymbol{w}_{\Gamma} \in \widehat{\boldsymbol{W}}_{\Gamma, B}$.

Proof. A proof using pointwise scaling can be found in [30,52]. By examining the arguments of those proofs, we find that it is enough to show that the scaling matrices (20) preserve the average of the normal flux over each subdomain face. This holds for our scaling matrices (20) by construction. Since each average acts on one face, and the nonzero entries of $B_{0 \Gamma}$ and $\widetilde{B}_{0 \Gamma}$ correspond to $\Pi_{F}$, i.e., the primal dof representing the average normal flux over $F$, we can conclude that

$$
B_{0 \Gamma} \widetilde{R}_{D, \Gamma}^{T} \mathbf{w}_{\Gamma}=\widetilde{B}_{0 \Gamma} \widetilde{R}_{\Gamma} \widetilde{R}_{D, \Gamma}^{T} \mathbf{w}_{\Gamma}=B_{0 \Pi} \mathbf{w}_{\Pi}=0
$$

Remark 3.6. A standard application of the deluxe scaling (cf. [14]) does not preserve the no-net-flux condition for the dual part of the vector, since the face scaling matrices will have a nonvanishing off-diagonal block $D_{\Pi_{F}^{c} \Pi_{F}}^{(i)}$. The scaling matrices given in (20) can be also used to design BDDC deluxe algorithms in the context of almost incompressible elasticity $[10,43]$ and incompressible Stokes flow; in these cases, depending on the discretization space, we will have additional $\Pi_{E}$ dofs on the subdomain edges that are involved in the enforcement of the no-net-flux condition; cf. [30, section 7].

We then seek a suitable splitting of the solution of (4) into a benign and a malign part. As proposed in [53, section 4.8.1], we can compute the malign part by using the 
action of the BDDC preconditioner (21) on a suitable right-hand side as

$$
\left[\begin{array}{c}
\mathbf{u}_{h, \Gamma}^{*} \\
\mathbf{p}_{h, 0}^{*}
\end{array}\right]=M_{\Gamma}^{-1}\left[\begin{array}{c}
0 \\
\mathbf{g}_{h, 0}^{*}
\end{array}\right]
$$

where $\mathbf{g}_{h, 0}$ is the $Q_{0}$ component of the right-hand side of (8). Discarding $\mathbf{p}_{h, 0}^{*}$, and denoting by $\mathbf{u}_{h}^{*}$ the discrete harmonic extension of $\mathbf{u}_{h, \Gamma}^{*}$, i.e., $\left(\mathbf{u}_{h}^{*}\right)_{I}=-K_{I I}^{-1} K_{I \Gamma} \mathbf{u}_{h, \Gamma}^{*}$, we split the solution of (4) into

$$
\left[\begin{array}{c}
\mathbf{u}_{h} \\
\mathbf{p}_{h}
\end{array}\right]=\left[\begin{array}{c}
\mathbf{u}_{h}^{*} \\
0
\end{array}\right]+\left[\begin{array}{c}
\overline{\mathbf{u}}_{h} \\
\mathbf{p}_{h}
\end{array}\right]
$$

where the correction satisfies

$$
\left[\begin{array}{cc}
A & B^{T} \\
B & -C
\end{array}\right]\left[\begin{array}{c}
\overline{\mathbf{u}}_{h} \\
\mathbf{p}_{h}
\end{array}\right]=\left[\begin{array}{l}
\mathbf{G}_{h}-A \mathbf{u}_{h}^{*} \\
\mathbf{g}_{h}-B \mathbf{u}_{h}^{*}
\end{array}\right]
$$

By construction, we have that $B_{0 \Gamma} \mathbf{u}_{h, \Gamma}^{*}=\mathbf{g}_{h, 0}$, provided that the dual space satisfies the no-net-flux condition (14); thus, $\overline{\mathbf{u}}_{h, \Gamma}$ lies in the benign subspace $\widehat{\boldsymbol{W}}_{\Gamma, B}$.

Remark 3.7. Divergence-free corrections can be considered by introducing a coarse triangulation and an associated div-conforming space $[15,52]$. Instead, our algorithm targets general subdomain distributions, as given by mesh partitioners, where regular coarse elements cannot be obtained by merging the elements of the fine mesh; however, we rely on the existence of a coarse triangulation in our analysis in section 4 .

3.5. Multilevel extensions. The subassembled nature of the coarse problem (24) naturally leads to a multilevel extension of the algorithm, where a subdomain at a finer level is considered an element of a coarser mesh; we note that these ideas were first presented in $[55,56]$ for a three-level algorithm, and then generalized to an arbitrary number of levels in [36]. Here we extend the three-level BDDC algorithm for saddle point problems introduced in [57] to an arbitrary number of levels.

In the presentation of the multilevel algorithm, we denote the finest level by $l=0$, and by $L$ the total number of levels. We denote by $K^{l}$ the matrix problem on level $l$, and set $K^{l+1}:=S_{C C}^{l}(0 \leq l<L-1)$ with $S_{C C}^{l}$ the coarse problem at level $l . K^{0}$ is given by (8), and the following split, in the spirit of (4), into velocity and pressure fields holds:

$$
K^{l}=\left[\begin{array}{cc}
A^{l} & B^{l^{T}} \\
B^{l} & 0
\end{array}\right], \quad l>0,
$$

where

$$
B^{l}=\left[\begin{array}{c}
B_{I}^{l} \\
B_{0}^{l}
\end{array}\right]=\left[\begin{array}{cc}
B_{I I}^{l} & B_{I \Gamma}^{l} \\
0 & B_{0 \Gamma}^{l}
\end{array}\right]
$$

When the no-net-flux condition is satisfied, we have $B_{0 \Gamma}^{l}=\left[\begin{array}{ll}0 & B_{0 \Pi}^{l}\end{array}\right]$ and $B^{l+1}=B_{0 \Pi}^{l}$; see [57, Lemma 4.1]. Note that a standard BDDC algorithm has $L=2$ levels; the coarse problem at level 0 is obtained by assembling coarse element contributions as detailed in (24), and it is solved exactly.

In a multilevel BDDC algorithm, the exact solution of the coarse problem at level $l$ is replaced by the application of a coarse BDDC preconditioner, denoted by $M_{l+1}^{-1}$, and which is of the form (13). In the multilevel framework, level $l$ subdomains are considered elements of level $l+1$; these can be aggregated into level $l+1$ subdomains 
by using unstructured mesh partitioning algorithms. We iterate this procedure until $l<L-1$, and denote by $M_{m l, L}^{-1}$ the resulting multilevel BDDC preconditioner. Such an approach requires less memory than a two-level method that uses a direct coarse solver, and it can lead to highly scalable algorithms (in a weak scaling sense), provided a suitable primal space for the coarser levels of the hierarchy is found. We note that, with the notation adopted, $M_{m l, 2}^{-1}$ denotes a standard two-level BDDC preconditioner.

Note that the subdomain matrices at level $l>0$ can be written as (cf. (5))

(a) $K^{l,(i)}=\left[\begin{array}{cc}A^{l,(i)} & B^{l,(i)^{T}} \\ B^{l,(i)} & 0\end{array}\right]$.

(b) $K^{l,(i)}=A^{l,(i)}$.

In case (a), the $i$ th subdomain at level $l$ contains any of the malign subdomains at level $l-1$. However, within the coarsening process, it may happen that just benign subdomains are aggregated; this case corresponds to (b). We define coarser subdomains as malign if the associated operator is of type (a), and the coarse constant pressure vector is in the kernel of the operator. We then construct $M_{l}^{-1}$ as outlined in the previous sections.

Theoretically, the condition number of multilevel BDDC methods depends multiplicatively on the condition numbers of the level problems as (cf. [36])

$$
\kappa\left(M_{m l, L}^{-1} K\right) \leq C \prod_{l=0}^{L-2} \kappa\left(M_{l}^{-1} K^{l}\right) .
$$

However, the coarsening ratio (i.e., the ratio between fine and coarse dofs) of twolevel BDDC methods are usually much larger than those needed to construct effective two-grid methods; therefore, we expect that multilevel BDDC will require many fewer levels than, e.g., multigrid methods, to reach a point where the coarsest problem can be efficiently solved by a direct method.

In addition, $\kappa\left(M_{m l}^{-1} K\right)$ can be controlled by finely tuning each of the product terms $\kappa\left(M_{l}^{-1} K^{l}\right)$, using adaptively constructed primal spaces that preserve the no-net-flux condition at each level of the hierarchy; for additional details, see section 4.1.2 and Theorem 4.3. We finally note that the rate of convergence of the resulting algorithm can be further accelerated by using few Chebyshev iterations at the coarser levels.

A three-level Darcy framework has been proposed in [57] when all subdomains are malign. The result of Lemma 4.2 in [57] is crucial to guarantee that the multilevel preconditioner $M_{m l, L}^{-1}$ keeps the conjugate gradient iterations in the benign subspace; that lemma naturally extends to our multilevel case, provided that the primal spaces satisfy the no-net-flux condition on each level. The quadrature weights, associated with the computation of the average normal flux over each face, and at each level of the hierarchy, can be computed by recursively projecting the $B$ block of the matrix given in (8) on the basis functions of the coarser velocity spaces; see also Remark 3.2.

As for the two-level BDDC algorithm, in order to use preconditioned conjugate gradients to solve (4), we need to split the solution into a benign and a malign part. One of the main contributions of our work to the multilevel Darcy theory is the efficient computation of the malign part of the solution $\mathbf{u}_{h}^{*}$ that we address in Algorithm 1; once $\mathbf{u}_{h}^{*}$ has been computed, we can solve (28) by using a preconditioned conjugate gradient method equipped with the multilevel BDDC preconditioner $M_{m l, L}^{-1}$.

LEMma 3.8. Let the primal spaces satisfy the no-net-flux condition on each level, and $\mathbf{u}_{h}^{*}$ be chosen by using Algorithm 1 . Then, $B_{0} \mathbf{u}_{h}^{*}=\mathbf{g}_{0, h}$. 


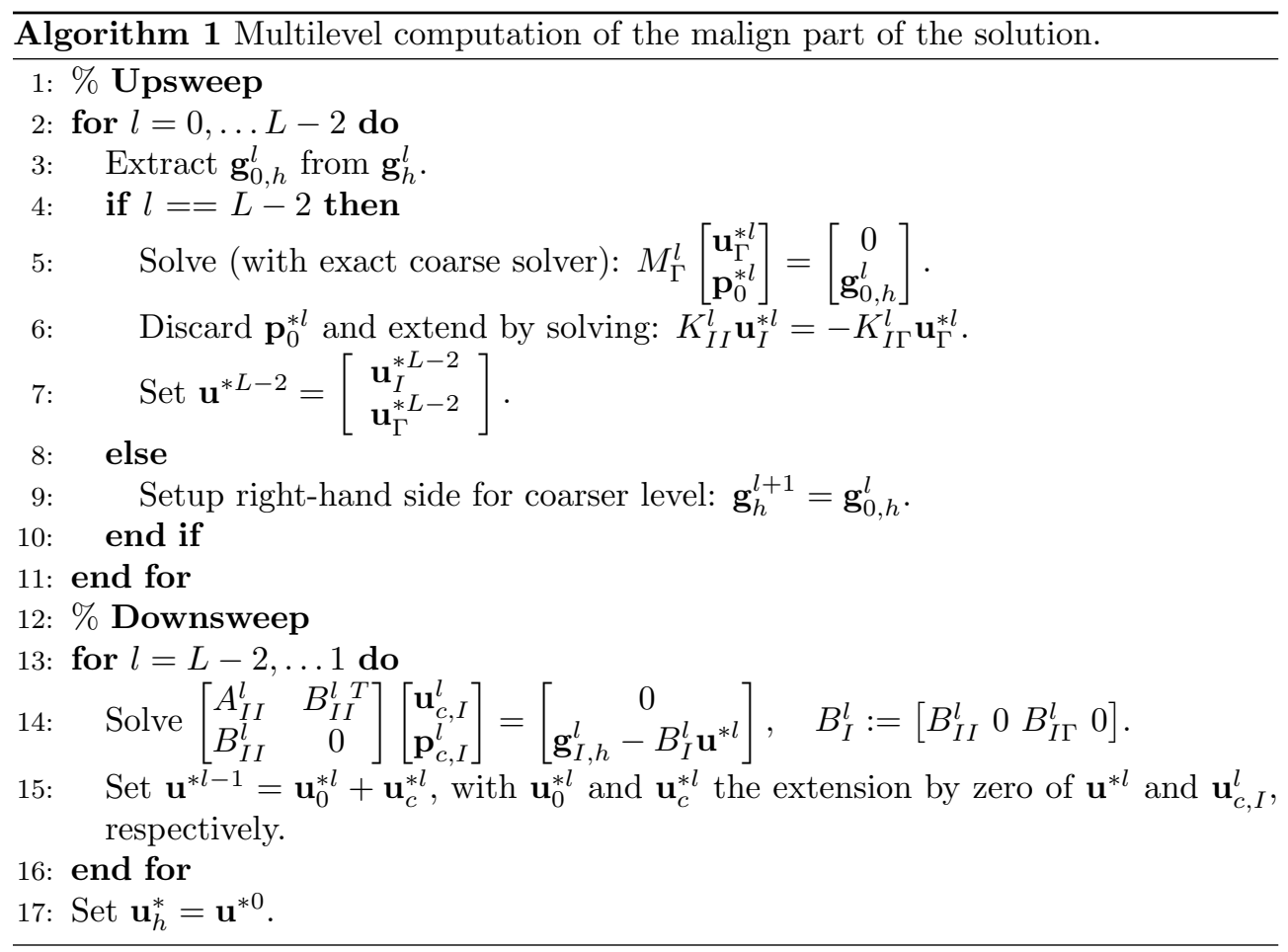

Proof. Clearly, we have that $B_{0} \mathbf{u}_{h}^{*}=\mathbf{g}_{0, h}$ if $L=2$, since Algorithm 1 reduces to the standard choice already presented in (26). We then proceed by induction, and assume that the lemma holds for $L=N$, and prove it for $N+1$. We have

$$
B_{0} \mathbf{u}_{h}^{*}=B^{1} \mathbf{u}^{* 1}+B^{1} \mathbf{u}_{c}^{* 1}=\left[\begin{array}{l}
B_{I}^{1} \mathbf{u}^{* 1}+B_{I}^{1} \mathbf{u}_{c}^{* 1} \\
B_{0}^{1} \mathbf{u}^{* 1}+B_{0}^{1} \mathbf{u}_{c}^{* 1}
\end{array}\right],
$$

where we have used the no-net-flux condition on the finest level and (29) for the first equation. Since $\mathbf{u}_{c}^{* 1}$ vanishes on the interface of level $1, B_{0}^{1} \mathbf{u}_{c}^{* 1}=0$. Moreover, we have by construction that $B_{I}^{1} \mathbf{u}^{* 1}+B_{I}^{1} \mathbf{u}_{c}^{* 1}=\mathbf{g}_{I, h}^{1}$. Since the induction assumption guarantees that $B_{0}^{1} \mathbf{u}^{* 1}=\mathbf{g}_{0, h}^{1}$, we have $B_{0} \mathbf{u}_{h}^{*}=\left[\begin{array}{l}\mathbf{g}_{I, h}^{1} \\ \mathbf{g}_{0, h}^{1}\end{array}\right]=\mathbf{g}_{0, h}$.

Remark 3.9. Similar ideas have been generalized to arbitrary numbers of levels in [47]. However, the multilevel BDDC proposed in [47] requires a divergence-free correction; there, the non-divergence-free part of the solution (i.e., $\mathbf{u}_{h}^{*}$ in our theory) is computed by solving, on each level, a linear system with the matrix $K^{l}$ and with $M^{l}$ as a preconditioner. The application of $M^{l}$ in turn involves a single upsweep and downsweep through the multilevel hierarchy, from level $l$ to $L-1$. Our algorithm instead requires a correction that belongs to $\widehat{\boldsymbol{W}}_{\Gamma, B}$; this allows us to use a more efficient procedure for the computation of $\mathbf{u}_{h}^{*}$, where just the coarsest problem has to be solved exactly (cf. line 5 in Algorithm 1), and only the interior problems have to be solved when downsweeping the hierarchy (cf. line 14 in Algorithm 1).

3.6. Preserving the complexity of the local solvers. The pressure change of basis is a fundamental step in our preconditioner, as it guarantees the well posedness of the interior problems (12) and of the Schur complements (9) for malign subdomains. 
However, using this basis change drastically limits the applicability of the algorithm since the memory required by the subdomain $\mathrm{LDL}^{\mathrm{T}}$ solvers becomes prohibitive, as a result of a drastic change in the sparsity pattern of the FE matrices. Indeed, this change of basis couples all the pressure dofs together, and thus spoils any possibility of having an optimal elimination tree of the unknowns.

We present a strategy which overcomes this problem by reusing solvers constructed for the original FE matrices. This approach consists of factoring these matrices after that one row and column, associated with a pressure dof, have been zeroed out, and by setting the corresponding diagonal entry to one. We can then construct the local solvers needed by the algorithm by using rank-one updates, without degrading the computational complexity of the factorizations. A different technique has been considered in [19, section 7.1.1] in order to avoid a pressure change of basis for a balancing Neumann-Neumann solver for almost incompressible elasticity.

For the same complexity reasonings, we avoid any change of basis on the velocity space before factoring the subdomain matrices; we will incorporate it at a later stage (see also Remark 4.2).

In order to keep the notation simple, in the following we drop the subdomain superscript, since all the algebra carried out in this section is local to each subdomain.

3.6.1. Avoid the pressure change of basis. Let $Z$ be the matrix defined in (5), obtained from the assembly of problem (1) on a subdomain $\Omega_{i}$, and let the unknowns be reordered into interior velocity first (subscript $v_{I}$ ), interior pressure $(p)$, and interface velocity last $\left(v_{\Gamma}\right)$. As is usually done in BDDC methods (see, e.g., [26]), our pressure change of basis can be performed by projection as

$$
\Xi^{T} Z \Xi, \quad \Xi=\left[\begin{array}{ccc}
I_{v_{I}} & 0 & 0 \\
0 & \Xi_{p} & 0 \\
0 & 0 & I_{v_{\Gamma}}
\end{array}\right]
$$

where $\Xi_{p}$ represents the change of basis on the pressure, from $Q_{I} \times Q_{0}$ to $P_{h}$, and it has the form

$$
\Xi_{p}=\left[\Xi_{p, I} \mid \Xi_{p, 0}\right], \quad \Xi_{p, I}=\left[\begin{array}{ccc}
1 & & \\
& \ddots & \\
& & 1 \\
-1 & \ldots & -1
\end{array}\right], \quad \Xi_{p, 0}=\mathbf{1}_{p} .
$$

$I_{v_{I}}$ and $I_{v_{\Gamma}}$ are identity matrices of appropriate sizes. The subdomain matrix (7) can be recast as

$$
\left[\begin{array}{cc}
\Xi_{I}^{T} Z_{I I} \Xi_{I} & \Xi_{I}^{T} Z_{I \Gamma} \\
Z_{I \Gamma}^{T} \Xi_{I} & Z_{\Gamma \Gamma}
\end{array}\right], \quad \Xi_{I}=\left[\begin{array}{cc}
I_{v_{I}} & 0 \\
0 & \Xi_{p, I}
\end{array}\right]
$$

where $Z_{I I}, Z_{I \Gamma}$, and $Z_{\Gamma \Gamma}$ are the submatrices of $Z$ corresponding to the splitting into interior and interface dofs. The matrix $Z_{I I}$ is singular with a kernel spanned by the vector which is 1 on the pressure dofs and 0 elsewhere.

We first design a solver for $K_{I I}=\Xi_{I}^{T} Z_{I I} \Xi_{I}$ in (13). Since $\operatorname{ker}\left(Z_{I I}\right) \perp \operatorname{range}\left(\Xi_{I}\right)$,

$$
\left(\Xi_{I}^{T} Z_{I I} \Xi_{I}\right)^{-1}=\left(\Xi_{I}^{T} \Xi_{I}\right)^{-1} \Xi_{I}^{T} Z_{I I}^{\dagger} \Xi_{I}\left(\Xi_{I}^{T} \Xi_{I}\right)^{-1},
$$

where the superscript $\dagger$ denotes pseudoinversion. Since the choice of a pseudoinverse is not unique, we can choose to annihilate the last (in the subdomainwise ordering) pressure dof by using

$$
Z_{I I}^{\dagger}:=\left[\begin{array}{cc}
Z_{I I}^{*-1} & 0 \\
0 & 0
\end{array}\right]
$$


Denoting by $n_{p}$ the number of pressure dofs, and by $p^{*}$ the subset of the pressure dofs from which the one ordered last has been removed, we have

$$
\left(\Xi_{I}^{T} \Xi_{I}\right)^{-1}=\left[\begin{array}{cc}
I_{v_{I}} & 0 \\
0 & I_{p^{*}}+\mathbf{1}_{p^{*}} \mathbf{1}_{p^{*}}^{T}
\end{array}\right]^{-1}=\left[\begin{array}{cc}
I_{v_{I}} & 0 \\
0 & I_{p^{*}}-\frac{1}{n_{p}} \mathbf{1}_{p^{*}} \mathbf{1}_{p^{*}}^{T}
\end{array}\right],
$$

where we have used the Sherman-Morrison formula to derive the second equality. The action of $\Xi_{I}\left(\Xi_{I}^{T} \Xi_{I}\right)^{-1}$ (similarly of its transpose) can thus be computed as a rank-one update on the $p^{*}$ pressure dofs, since, for our choice of pseudoinverse (33), the value of the last pressure dof is always zero, and we can thus neglect its contribution.

Within deluxe BDDC algorithms, the explicit knowledge of the local Schur complement $S_{\Gamma}(9)$ is required for efficient adaptive algorithms [63] (see also section 4.1); by using (31) and (32), we find that

$$
S_{\Gamma}=Z_{\Gamma \Gamma}-Z_{I \Gamma}^{T} \Xi_{I}\left(\Xi_{I}^{T} \Xi_{I}\right)^{-1} \Xi_{I}^{T} Z_{I I}^{\dagger} \Xi_{I}\left(\Xi_{I}^{T} \Xi_{I}\right)^{-1} \Xi_{I}^{T} Z_{I \Gamma}
$$

A direct computation reveals

$$
\Xi_{I}\left(\Xi_{I}^{T} \Xi_{I}\right)^{-1} \Xi_{I}^{T}=\left[\begin{array}{ccc}
I_{v_{I}} & 0 & 0 \\
0 & r_{p^{*}} & 0 \\
0 & 0 & 0
\end{array}\right]-\frac{1}{n_{p}}\left[\begin{array}{ccc}
0 & 0 & 0 \\
0 & X & \mathbf{1}_{p^{*}} \\
0 & \mathbf{1}_{p^{*}}^{T} & 1-n_{p}
\end{array}\right],
$$

where $X=\left[\mathbf{1}_{p^{*}}|\cdots| \mathbf{1}_{p^{*}}\right]$. With the chosen pseudoinverse (33), we can ignore the contribution from the last row of the second matrix and obtain, using $\alpha:=1 / n_{p}$,

$$
S_{\Gamma}=S_{1}+\alpha\left(S_{2}+S_{2}^{T}\right)-\alpha^{2} S_{3}, \quad S_{2}=Z_{I \Gamma}^{T} M^{T} Z_{I I}^{\dagger} I^{*} Z_{I \Gamma}, \quad S_{3}=Z_{I \Gamma}^{T} M^{T} Z_{I I}^{\dagger} M Z_{I \Gamma},
$$

with

$$
I^{*}=\left[\begin{array}{rrr}
I_{v_{I}} & 0 & 0 \\
0 & I_{p^{*}} & 0 \\
0 & 0 & 0
\end{array}\right], \quad M=\left[\begin{array}{ccc}
0 & 0 & 0 \\
0 & X & 1_{p^{*}} \\
0 & 0 & 0
\end{array}\right] .
$$

$S_{1}$ is the Schur complement of the matrix $Z^{*}$, obtained after removing from $Z$ the row and column associated with the last pressure dof, and by setting to one the corresponding diagonal entry. Note that $S_{1}$ can be explicitly obtained by using the Schur complement feature of any of the numerical factorization packages MUMPS [1], PASTIX [20], and PARDISO [27].

Let $s$ be the sum of the $p^{*}$ entries of the vector $\mathbf{v}^{*}=Z_{I I}^{*-1} \mathbf{1}^{*}$ with $\mathbf{1}^{*}$ the extension by zero of $\mathbf{1}_{p^{*}}$; in addition, let $\mathbf{c}_{\Gamma}$ be the vector whose entries are the sum of the columns of $Z_{I \Gamma}$ restricted to the pressure; the contributions of $S_{2}$ and $S_{3}$ to $S_{\Gamma}$ are rank-one updates given by $S_{2}=Z_{I \Gamma}^{* T} \mathbf{v}^{*} c_{\Gamma}^{T}$ and $S_{3}=s \mathbf{c}_{\Gamma} \mathbf{c}_{\Gamma}^{T}$.

The application of the preconditioner (21) requires a solver for the subdomain problem (31); see [12]. By reusing $S_{\Gamma}$ given in (34), we can proceed as follows.

1. Correct the interior right-hand side: $\widetilde{\mathbf{g}}_{I}=\Xi_{I}\left(\Xi_{I}^{T} \Xi_{I}\right)^{-1} \mathbf{g}_{I}$.

2. Solve the interior problem: $\mathbf{b}_{I}=Z_{I I}^{*-1} \widetilde{\mathbf{g}}_{I}$.

3. Obtain a right-hand side for the Schur complement: $\mathbf{b}_{\Gamma}=\mathbf{g}_{\Gamma}-Z_{I \Gamma}^{* T} \mathbf{b}_{I}$.

4. Compute a correction for the Schur right-hand side: $\mathbf{b}_{\Gamma}^{*}=-Z_{I \Gamma}^{T} M^{T} Z_{I I}^{*-1} \widetilde{\mathbf{g}}_{I}$.

5. Solve with the Schur complement: $S_{\Gamma} \mathbf{x}_{\Gamma}=\mathbf{b}_{\Gamma}+\mathbf{b}_{\Gamma}^{*}$.

6. Extend into the interior: $\widetilde{\mathbf{x}}_{I}=\mathbf{b}_{I}-Z_{I \Gamma}^{*} \mathbf{x}_{\Gamma}$.

7. Compute a correction for the Schur solution: $\mathbf{x}_{I}^{*}=-Z_{I I}^{*-1} M Z_{I \Gamma} \mathbf{x}_{\Gamma}$.

8. Correct the interior solution: $\mathbf{x}_{I}=\Xi_{I}\left(\Xi_{I}^{T} \Xi_{I}\right)^{-1}\left(\widetilde{\mathbf{x}}_{I}+\mathbf{x}_{I}^{*}\right)$.

The action of the matrix needed in steps 4 and 7 can be obtained with a rankone update operation as $Z_{I I}^{*-1} M Z_{I \Gamma}=-\frac{1}{n_{p}} \overline{\mathbf{v}}^{*} \mathbf{c}^{T}$, where $\overline{\mathbf{v}}^{*}$ is the same as $\mathbf{v}^{*}$ except that its last pressure dof vanishes. We remark that the backward substitution steps 
$(2,3$, and 6$)$ are performed within the factorization package used in the numerical experiments; the complete procedure is thus optimal from the memory viewpoint, as it just needs 4 rank-one updates (steps 1, 4, 7, and 8) in addition to the backward substitutions and the solution of the Schur complement equations.

3.6.2. Avoid the velocity change of basis. Explicit primal dofs are not needed in practical implementations of the BDDC algorithm; cf. [12, section 4]. However, for our algorithm to succeed, it is mandatory that the scaling preserve the net flux across subdomain boundaries (see Lemma 3.5). To this end, we note that we can change the basis on the velocity space on the fly, before applying the scaling; a change of basis is still needed for the small and dense scaling matrices.

For simplicity, we omit the subdomain index $(i)$ here. Let $\Psi_{F}$ represent an orthonormal change of basis for the dofs on $F$ that reveals the average normal flux over the face; we note that this matrix can be constructed using a $\mathrm{QR}$ decomposition of the quadrature weights $\mathbf{q}_{F}$ associated with (15) as

$$
\Psi_{F}=\left[\begin{array}{ll}
Q_{r} \widetilde{R}^{-T} & Q_{k}
\end{array}\right], \quad \mathbf{q}_{F}=Q R, \quad Q=\left[\begin{array}{ll}
Q_{r} & Q_{k}
\end{array}\right], \quad R=\left[\begin{array}{c}
\widetilde{R} \\
0
\end{array}\right]
$$

where subscripts $r$ and $k$ denote range and kernel of $\mathbf{q}_{F}$. The scaling matrix $D_{F}$ for the dofs of $F$ is then performed as $\Psi_{F} D_{F}^{*} \Psi_{F}^{T}$, where $D_{F}^{*}$ is assembled as in (20), by using the projections of the original principal minors of S, i.e., $\Psi_{F}^{T}\left(R_{F} S_{\Gamma} R_{F}^{T}\right) \Psi_{F}$, after having put to zero the row and column related to the explicit primal dof representing the average normal flux over $F$, and by setting to one the corresponding diagonal entry.

We next show how to obtain the subdomain contribution to the coarse problem matrix given in (25). The leading block of $S_{\Pi \Pi}$ can be computed as in conventional BDDC methods [12, section 4], while the off-diagonal block $B_{0 \Pi}$ can be obtained by projecting the subdomain matrix $B$ of (5) onto the subdomain component of $\boldsymbol{W}_{\Pi} \times Q_{0}$ as $\Phi^{T} B^{T} \mathbf{1}$ with $\mathbf{1}$ a constant unit vector of appropriate size, and $\Phi$ the matrix of coarse basis functions defined in (23).

4. Theoretical estimates. In this section, we provide the condition number estimates for the BDDC preconditioned operator with both the minimal and adaptive coarse spaces. We introduce an average operator and the condition number bound is first reduced to a bound for the average operator. The average operator is then estimated using a face lemma for the minimal coarse space and by the construction of the coarse space for the adaptive coarse space.

4.1. Condition number bounds. We define the average operator

$$
E_{D}:=\widetilde{R}_{\Gamma} \widetilde{R}_{D, \Gamma}^{T}
$$

and we use the notation $\|\boldsymbol{w}\|_{A}^{2}:=\mathbf{w}^{T} A \mathbf{w}$. From now on, $\boldsymbol{w}$ denotes a function belonging to $\widetilde{\boldsymbol{W}}_{\Gamma, B}$ with $\boldsymbol{w}^{(i)}=\widetilde{R}_{\Gamma}^{(i)} \boldsymbol{w} \in \boldsymbol{W}_{\Gamma}^{(i)}$; the restriction of $\boldsymbol{w}^{(i)}$ to a face $F \subset \partial \Omega_{i}$ is denoted by $\boldsymbol{w}_{F}^{(i)}$.

Theorem 4.1 (see [30, Theorem 1]). Let the dual space satisfy the no-net-flux condition (14). Then, $M_{\Gamma}^{-1} \widehat{S}$ is symmetric positive definite on the subspace $\widehat{\boldsymbol{W}}_{\Gamma, B} \times$ $Q_{0}$. The minimum eigenvalue is 1 and the maximum eigenvalue is bounded from above by $\left\|E_{D}\right\|_{\widetilde{S}_{\Gamma}}^{2}$ with the norm taken on $\widetilde{\boldsymbol{W}}_{\Gamma, B}$. 
Instead of developing an estimate for $E_{D}$, we work with the complementary projector $P_{D}=I-E_{D}$, since the two operators have the same $\widetilde{S}_{\Gamma}$-norm [34]. Thus, we estimate $\left\|R_{F}^{(i) T}\left(\boldsymbol{w}_{F}^{(i)}-\overline{\boldsymbol{w}}_{F}\right)\right\|_{S_{\Gamma}^{(i)}}^{2}$, where $\overline{\boldsymbol{w}}_{F}=\left(E_{D} \boldsymbol{w}\right)_{F}=D_{F}^{(i) T} \mathbf{w}_{F}^{(i)}+D_{F}^{(j) T} \mathbf{w}_{F}^{(j)}$ with $D_{F}^{(i)}$ given by $(20)$. We can rewrite $\left\|R_{F}^{(i) T}\left(\boldsymbol{w}_{F}^{(i)}-\overline{\boldsymbol{w}}_{F}\right)\right\|_{S_{\Gamma}^{(i)}}^{2}$ as

$$
\left(\mathbf{w}_{F}^{(i)}-\mathbf{w}_{F}^{(j)}\right)^{T} D_{F}^{(j)} S_{F F}^{(i)} D_{F}^{(j) T}\left(\mathbf{w}_{F}^{(i)}-\mathbf{w}_{F}^{(j)}\right),
$$

where $S_{F F}^{(i)}$ is given by (19). Adding the contribution from subdomain $j$, we obtain

$$
\left(\mathbf{w}_{F}^{(i)}-\mathbf{w}_{F}^{(j)}\right)^{T}\left(\bar{S}_{F F}^{(i)}: \bar{S}_{F F}^{(j)}\right)\left(\mathbf{w}_{F}^{(i)}-\mathbf{w}_{F}^{(j)}\right), \quad \bar{S}_{F F}^{(i)}:=\left[\begin{array}{cc}
S_{\Pi_{F}^{c} \Pi_{F}^{c}}^{(i)} & 0 \\
0 & S_{\Pi_{F} \Pi_{F}}^{(i)}
\end{array}\right],
$$

where $A: B:=\left(A^{-1}+B^{-1}\right)^{-1}$ denotes the parallel sum of two symmetric positive definite matrices; see [2]. Since $\mathbf{w}_{F}^{(i)}-\mathbf{w}_{F}^{(j)}$ vanishes on $\Pi_{F}, S_{\Pi_{F} \Pi_{F}}^{(i)}$ and $S_{\Pi_{F} \Pi_{F}}^{(j)}$ are irrelevant.

4.1.1. Minimal coarse space. We can bound (37) by $2\left(\mathbf{w}_{F}^{(i)}-\mathbf{w}_{\Pi_{F}}\right)^{T}\left(\bar{S}_{F F}^{(i)}: \bar{S}_{F F}^{(j)}\right)\left(\mathbf{w}_{F}^{(i)}-\mathbf{w}_{\Pi_{F}}\right)+2\left(\mathbf{w}_{F}^{(j)}-\mathbf{w}_{\Pi_{F}}\right)^{T}\left(\bar{S}_{F F}^{(i)}: \bar{S}_{F F}^{(j)}\right)\left(\mathbf{w}_{F}^{(j)}-\mathbf{w}_{\Pi_{F}}\right)$ with $\mathbf{w}_{\Pi_{F}}$ the average normal flux of $\mathbf{w}_{F}^{(i)}$ and $\mathbf{w}_{F}^{(j)}$ over $F$. Using $A: B \leq A$ (in the sense of quadratic forms), and the fact that $\left(\mathbf{w}_{F}^{(i, j)}-\mathbf{w}_{\Pi_{F}}\right)_{\Pi_{F}}=0$, the latter expression can be further bounded by

$$
2\left(\mathbf{w}_{F}^{(i)}-\mathbf{w}_{\Pi_{F}}\right)^{T} S_{F F}^{(i)}\left(\mathbf{w}_{F}^{(i)}-\mathbf{w}_{\Pi_{F}}\right)+2\left(\mathbf{w}_{F}^{(j)}-\mathbf{w}_{\Pi_{F}}\right)^{T} S_{F F}^{(j)}\left(\mathbf{w}_{F}^{(j)}-\mathbf{w}_{\Pi_{F}}\right) .
$$

We are then left to bound the first term in (38) by the energy of any benign extension of the dofs $\mathbf{w}_{F}^{(i)}$ to the rest of $\Gamma_{i}$ using a face lemma (see Lemmas 4.7 and 4.8 ); the second term, corresponding to subdomain $j$, can be treated similarly. Our main result for the minimal coarse space is given in Theorem 4.9.

4.1.2. An adaptive coarse space. For an adaptive BDDC deluxe algorithm, a face lemma is replaced by a generalized eigenvalue computation. Following [44], we consider energy minimizing extensions and introduce the matrix

$$
\widetilde{S}_{\Pi_{F}^{c} \Pi_{F}^{c}}^{(i)}=S_{\Pi_{F}^{c} \Pi_{F}^{c}}^{(i)}-S_{F^{0} \Pi_{F}^{c}}^{(i) T} S_{F^{0} F^{0}}^{(i)-1} S_{F^{0} \Pi_{F}^{c}}^{(i)},
$$

where $F^{0}=\Gamma_{i} \backslash \Pi_{F}^{c}, S_{F^{0} F^{0}}^{(i)}$, the associated principal minor of $S_{\Gamma}^{(i)}$, and $S_{F^{0} F^{*}}^{(i)}$, a corresponding off-diagonal block. We then consider the generalized eigenvalue problem

$$
\widetilde{S}_{\Pi_{F}^{c} \Pi_{F}^{c}}^{(i)}: \widetilde{S}_{\Pi_{F}^{c} \Pi_{F}^{c}}^{(j)} \psi=\nu S_{\Pi_{F}^{c} \Pi_{F}^{c}}^{(i)}: S_{\Pi_{F}^{c} \Pi_{F}^{c}}^{(j)} \psi
$$

The elements of the primal space are obtained as $S_{\Pi_{F}^{c} \Pi_{F}^{c}}^{(i)}: S_{\Pi_{F}^{c} \Pi_{F}^{c}}^{(j)} \Psi$, where $\Psi$ is the matrix formed columnwise by the eigenvectors of (40) with eigenvalues smaller than a fixed tolerance $1 / \nu_{t o l}$. We then extend these vectors by zero on $\Pi_{F}$, since we require that the adaptive primal basis have zero net flux over $\Gamma_{i}$. Using [50, Lemma 2.11] (see also [25, Lemma 4.6]), and the energy minimizing property of $\widetilde{S}_{\Pi_{F}^{c} \Pi_{F}^{c}}^{(i)}$ with respect to $S_{\Gamma}^{(i)}$, we can bound (37) by

(41) $2 \nu_{t o l}\left(\mathbf{w}_{\Pi_{F}^{c}}^{(i) T} \widetilde{S}_{\Pi_{F}^{c} \Pi_{F}^{c}}^{(i)} \mathbf{w}_{\Pi_{F}^{c}}^{(i)}+\mathbf{w}_{\Pi_{F}^{c}}^{(j) T} \widetilde{S}_{\Pi_{F}^{c} \Pi_{F}^{c}}^{(j)} \mathbf{w}_{\Pi_{F}^{c}}^{(j)}\right) \leq 2 \nu_{t o l}\left(\left\|\boldsymbol{w}^{(i)}\right\|_{S_{\Gamma}^{(i)}}^{2}+\left\|\boldsymbol{w}^{(j)}\right\|_{S_{\Gamma}^{(j)}}^{2}\right)$. 
Remark 4.2. For computational efficiency, the matrices defined in (39) are never explicitly computed. Instead, we can work with the principal minors of the inverse of $S_{\Gamma}^{(i)}[63]$. In our algorithm, we first invert the Schur complement given in (34), and we then consider a change of basis of its principal minors, relative to each face, by reusing the orthonormal matrices $\Psi_{F}$ defined in (35). Since the change of variables for all of $\Gamma_{i}$ is block-diagonal, we obtain $\widetilde{S}_{\Pi_{F}^{c} \Pi_{F}^{c}}^{(i)-1}$ by discarding the row and column that correspond to $\Pi_{F}$. Once the elements of the primal space have been computed, they are mapped to the original basis by left-multiplication with $\Psi_{F}$.

When using more general scaling techniques, we can consider the following eigenvalue problem (cf. [25, Lemma 4.10])

$$
\widetilde{S}_{\Pi_{F}^{c} \Pi_{F}^{c}}^{(i)}: \widetilde{S}_{\Pi_{F}^{c} \Pi_{F}^{c}}^{(j)} \psi=\nu Q \psi, \quad Q=D_{\Pi_{F}^{c}}^{(j) T} S_{\Pi_{F}^{c} \Pi_{F}^{c}}^{(i)} D_{\Pi_{F}^{c}}^{(j)}+D_{\Pi_{F}^{c}}^{(i) T} S_{\Pi_{F}^{c} \Pi_{F}^{c}}^{(j)} D_{\Pi_{F}^{c}}^{(i)} .
$$

Elements of the primal space are generated as $Q \Psi$, where $\Psi$ is the matrix of eigenvectors for which the eigenvalues are smaller than $1 / \nu_{t o l}$. The effects of using primal spaces generated by the deluxe and by the cardinality scaling are numerically studied in section 5 .

We close this section with the following theorem.

TheOrem 4.3 (adaptive BDDC deluxe). Let the assumptions of Theorem 4.1 hold. In addition, let the primal space contain all the eigenvectors of the generalized eigenvalue problems (40) or (42), whose corresponding eigenvalues are lower than $1 / \nu_{\text {tol }}$ for each $F \in \Gamma$. Then, the preconditioned BDDC operator for (4) satisfies

$$
\kappa\left(M^{-1} K\right)=\kappa\left(M_{\Gamma}^{-1} \widehat{S}\right) \leq C \nu_{t o l},
$$

where $C$ independent of $N, h, H$, and the coefficients of $K_{p}$ and $w$.

Proof. The left-hand term of (37) provides an upper bound of the square of the $S_{\Gamma}^{(i)}$-norm of a face component of the average operator; in a deluxe algorithm, it can be further bounded using the right-hand side of (41). Using Theorem 4.1, we obtain our result. Similar reasonings hold true with more general scaling techniques. The constant $C$ depends on $N_{F}^{2}$, where $N_{F}$ is the maximum number of faces of any subdomain. For additional details on the proof, see [8, 23, 25, 41, 45].

4.2. Technical tools and theoretical results. In order to develop our theory, we assume that each subdomain is a union of shape-regular coarse triangles, with diameter $H_{i}$, and that the number of coarse triangles forming an individual subdomain is uniformly bounded. Let $\boldsymbol{W}^{H}$ and $Q^{H}$ be the lowest order RT and pressure FE spaces defined on the coarse triangulation $\mathcal{T}_{H}$. For the sake of simplicity, we assume that $\partial \Omega_{D}=\emptyset$; we further assume that $w$ is constant in each subdomain, while we allow arbitrarily large coefficient jumps aligned with $\Gamma$, and denote by $w_{i}$ the value of $w$ on $\Omega_{i}$. We also allow aligned jumps in the coefficients of the permeability tensor $K_{p}$, denoting by $k_{i, M}$ and $k_{i, m}$ the largest and smallest eigenvalue of $K_{p}$ on $\Omega_{i}$. Finally, we use the abbreviation $C_{H, h}:=\max _{i}\left\{1+\log \left(H_{i} / h_{i}\right)\right\}$.

Given $\boldsymbol{u} \in H\left(\operatorname{div}, \Omega_{i}\right)$, it is possible to define its normal component $\boldsymbol{u} \cdot \boldsymbol{n}$ on $\partial \Omega_{i}$ as an element of $H^{-\frac{1}{2}}\left(\partial \Omega_{i}\right)$, and the following inequality holds (see [51, (10.40)]):

$$
\|\boldsymbol{u} \cdot \boldsymbol{n}\|_{H^{-\frac{1}{2}}\left(\partial \Omega_{i}\right)}^{2} \leq C\left(\|\boldsymbol{u}\|_{L^{2}\left(\Omega_{i}\right)}^{2}+H_{i}^{2}\|\nabla \cdot \boldsymbol{u}\|_{L^{2}\left(\Omega_{i}\right)}^{2}\right)
$$

with a constant $C$ that is independent of $H_{i}$. The operator that maps a vector in $H\left(\operatorname{div}, \Omega_{i}\right)$ into its normal component in $H^{-\frac{1}{2}}\left(\Omega_{i}\right)$ is thus continuous, and it can be shown to be surjective; see [18, Chap. I, Thm. 2.5, and Cor. 2.8]. 
We consider the interpolation operator $\Pi_{R T}^{H}$ from $\boldsymbol{U}_{h}$ to $\boldsymbol{W}^{H}$, defined by

$$
\lambda_{F}\left(\Pi_{R T}^{H} \boldsymbol{u}\right):=\frac{1}{|F|} \int_{F} \boldsymbol{u} \cdot \boldsymbol{n} d s, \quad F \subset F_{H},
$$

where $F_{H}$ is the set of element faces in the coarse triangulation $\mathcal{T}_{H}$. The stability of $\Pi_{R T}^{H}$ is established in the next lemma; cf. [62, Lemma 4.1].

Lemma 4.4. There exists a constant $C$, depending only on the aspect ratios of the elements of $\mathcal{T}_{H}$ and $\mathcal{T}_{h}$, such that, $\forall \mathcal{K} \in \mathcal{T}_{H}$ and $\forall \boldsymbol{u} \in \boldsymbol{U}_{h}$,

$$
\begin{aligned}
\left\|\nabla \cdot\left(\Pi_{R T}^{H} \boldsymbol{u}\right)\right\|_{L^{2}(\mathcal{K})}^{2} & \leq\|\nabla \cdot \boldsymbol{u}\|_{L^{2}(\mathcal{K})}^{2}, \\
\left\|\Pi_{R T}^{H} \boldsymbol{u}\right\|_{L^{2}(\mathcal{K})}^{2} & \leq C C_{H, h}\left(\|\boldsymbol{u}\|_{L^{2}(\mathcal{K})}^{2}+H_{\mathcal{K}}^{2}\|\nabla \cdot \boldsymbol{u}\|_{L^{2}(\mathcal{K})}^{2}\right) .
\end{aligned}
$$

We define $N\left(\partial \Omega_{i}\right)$ as the space of functions that are constant on each face of $\partial \Omega_{i}$, and its subspace $N_{0}\left(\partial \Omega_{i}\right)$, consisting of functions that have vanishing mean value over $\partial \Omega_{i}$. Let $N^{H}$ be the space of functions $\mu$ defined on $\Gamma$, such that for each subdomain $\Omega_{i}$ and each face $F$ of $\Omega_{i}, \mu$ is constant on $F ; N^{H}$ is the space of normal components on $\Gamma$ of vectors in $\boldsymbol{W}^{H}$.

The stable extension operator, defined in the next lemma, provides a divergencefree extension of boundary data given on $\partial \Omega_{i}$; cf. [62, Lemma 4.3]. Here, we use $\boldsymbol{W}^{(i)}:=\boldsymbol{W}_{I}^{(i)} \oplus \boldsymbol{W}_{\Gamma}^{(i)}$ to denote the local velocity space.

Lemma 4.5. There exists an extension operator $\tilde{\mathcal{H}}_{i}: N_{0}\left(\partial \Omega_{i}\right) \rightarrow \boldsymbol{W}^{(i)}$, such that

$$
\begin{aligned}
\nabla \cdot \tilde{\mathcal{H}}_{i} \mu & =0 \quad \text { for } x \in \Omega_{i}, \\
\left\|\tilde{\mathcal{H}}_{i} \mu\right\|_{L^{2}\left(\Omega_{i}\right)} & \leq C\|\mu\|_{H^{-\frac{1}{2}}\left(\partial \Omega_{i}\right)}
\end{aligned}
$$

for any $\mu \in N_{0}\left(\partial \Omega_{i}\right)$ with $C$ independent of $h, H$, and $\mu$.

Given a subdomain $\Omega_{i}$, we define a partition of unity functions associated with its faces. Let $\zeta_{F}$ be the function that is 1 on $F$ and 0 on $\partial \Omega_{i} \backslash F$. Given a function $\mu \in N\left(\partial \Omega_{i}\right)$ and a face $F \subset \partial \Omega_{i}$, let $\mu_{F}:=\zeta_{F} \mu \in N\left(\partial \Omega_{i}\right)$. We have the following estimate; cf. [62, Lemma 4.4].

Lemma 4.6. Let $\mu \in N_{0}\left(\partial \Omega_{i}\right)$ and, for any $F \subset \partial \Omega_{i}$, let $\int_{F} \mu d s=\int_{F} \mu_{F} d s=0$. There exists a constant $C$, independent of $h, H$, and $\mu_{H}$, such that, for any $\mu_{H} \in N^{H}$,

$$
\left\|\mu_{F}\right\|_{H^{-\frac{1}{2}}\left(\partial \Omega_{i}\right)}^{2} \leq C C_{H, h}\left(C_{H, h}\left\|\mu+\mu_{H}\right\|_{H^{-\frac{1}{2}}\left(\partial \Omega_{i}\right)}^{2}+\|\mu\|_{H^{-\frac{1}{2}}\left(\partial \Omega_{i}\right)}^{2}\right) .
$$

Let $\boldsymbol{w}_{\Pi_{F}}:=\Pi_{R T}^{H} \boldsymbol{w}_{F}^{(i)}$. We estimate the expression in (38) using the two following lemmas.

Lemma 4.7. For a malign subdomain $\Omega_{i}$, we have

$$
\left\|R_{F}^{T}\left(\boldsymbol{w}_{F}^{(i)}-\boldsymbol{w}_{\Pi_{F}}\right)\right\|_{S_{\Gamma}^{(i)}}^{2} \leq C \frac{k_{i, M}}{k_{i, m}} C_{H, h}^{2}\left\|\boldsymbol{w}^{(i)}\right\|_{S_{\Gamma}^{(i)}}^{2},
$$

where $C$ is independent of $N, h, H$, and the coefficient of $K_{p}$.

Proof. Let $\mathcal{E}_{i}: N_{0}(\partial \Omega) \rightarrow \boldsymbol{W}^{(i)}$ be the discrete Darcy extension operator, i.e.,

$$
\mathbf{a}_{i}\left(\mathcal{E}_{i} \boldsymbol{g}_{\Gamma}, \mathcal{E}_{i} \boldsymbol{g}_{\Gamma}\right):=\min _{\mathbf{g} \cdot \boldsymbol{n}=\mathbf{g}_{\Gamma} \cdot \boldsymbol{n}, \nabla \cdot \mathbf{g}=0} \mathbf{a}_{i}(\mathbf{g}, \mathbf{g}), \quad \mathbf{g} \in \boldsymbol{W}^{(i)},
$$

and let $\boldsymbol{v}:=\mathcal{E}_{i} \zeta_{F}\left(\boldsymbol{w}^{(i)}-\boldsymbol{w}_{\Pi_{F}}\right)$. Notice that $\left\|R_{F}^{T}\left(\boldsymbol{w}_{F}^{(i)}-\boldsymbol{w}_{\Pi_{F}}\right)\right\|_{S_{\Gamma}^{(i)}}^{2}=\mathbf{a}_{i}(\boldsymbol{v}, \boldsymbol{v})$.

Copyright $@$ by SIAM. Unauthorized reproduction of this article is prohibited. 
Since $\zeta_{F}\left(\boldsymbol{w}^{(i)}-\boldsymbol{w}_{\Pi_{F}}\right) \cdot \boldsymbol{n}$ has a vanishing mean value on $\partial \Omega_{i}$, letting $\boldsymbol{r}:=$ $\tilde{\mathcal{H}}_{i}\left(\zeta_{F}\left(\boldsymbol{w}^{(i)}-\boldsymbol{w}_{\Pi_{F}}\right) \cdot \boldsymbol{n}\right)$ and using Lemma 4.5 , we have $\nabla \cdot \mathbf{r}=0$ and

$$
\mathbf{a}_{i}(\boldsymbol{v}, \boldsymbol{v}) \leq \mathbf{a}_{i}(\boldsymbol{r}, \boldsymbol{r}) \leq k_{i, M}\|\boldsymbol{r}\|_{L^{2}\left(\Omega_{i}\right)}^{2} \leq C k_{i, M}\left\|\zeta_{F}\left(\boldsymbol{w}^{(i)}-\boldsymbol{w}_{\Pi_{F}}\right) \cdot \boldsymbol{n}\right\|_{H^{-\frac{1}{2}}\left(\partial \Omega_{i}\right)}^{2} .
$$

Since $\boldsymbol{w}$ is in the benign space, $\boldsymbol{w}^{(i)} \cdot \boldsymbol{n}$ has a vanishing mean value on $\partial \Omega_{i}$, and we can define $\mathcal{E}_{i} \boldsymbol{w}^{(i)}$. Let $\boldsymbol{u}_{H}:=\Pi_{R T}^{H}\left(\mathcal{E}_{i} \boldsymbol{w}^{(i)}\right)$ and $\mu_{H}:=\boldsymbol{u}_{H} \cdot \boldsymbol{n}$. By the definition of $\Pi_{R T}^{H}$, we have $\zeta_{F} \mu_{H}=\boldsymbol{w}_{\Pi_{F}} \cdot \boldsymbol{n}$ and $\int_{F}\left(\boldsymbol{w}^{(i)} \cdot \boldsymbol{n}-\mu_{H}\right) d s=0 \forall F \subset \partial \Omega_{i}$.

Using Lemma 4.6 and the triangle inequality, we have

$$
\left\|\zeta_{F}\left(\boldsymbol{w}^{(i)} \cdot \boldsymbol{n}-\mu_{H}\right)\right\|_{H^{-\frac{1}{2}}\left(\partial \Omega_{i}\right)}^{2} \leq C C_{H, h}\left(C_{H, h}\left\|\boldsymbol{w}^{(i)} \cdot \boldsymbol{n}\right\|_{H^{-\frac{1}{2}}\left(\partial \Omega_{i}\right)}^{2}+\left\|\mu_{H}\right\|_{H^{-\frac{1}{2}\left(\partial \Omega_{i}\right)}}^{2}\right) .
$$

By Lemma 4.4, we know that

$$
\begin{aligned}
\left\|\nabla \cdot \mathbf{u}_{H}\right\|_{L^{2}\left(\Omega_{i}\right)}^{2} & \leq\left\|\nabla \cdot \mathcal{E}_{i} \boldsymbol{w}^{(i)}\right\|_{L^{2}\left(\Omega_{i}\right)}^{2}=0, \\
\left\|\boldsymbol{u}_{H}\right\|_{L^{2}\left(\Omega_{i}\right)}^{2} & \leq C C_{H, h}\left\|\mathcal{E}_{i} \boldsymbol{w}^{(i)}\right\|_{L^{2}\left(\Omega_{i}\right)}^{2} .
\end{aligned}
$$

Using (45), (46), (44), and (47), we finally obtain

$$
\begin{aligned}
\left\|R_{F}^{T}\left(\boldsymbol{w}_{F}^{(i)}-\boldsymbol{w}_{\Pi_{F}}\right)\right\|_{S_{\Gamma}^{(i)}}^{2} & \leq C k_{i, M} C_{H, h}\left(C_{H, h}\left\|\mathcal{E}_{i} \boldsymbol{w}^{(i)}\right\|_{L^{2}\left(\Omega_{i}\right)}^{2}+\left\|\boldsymbol{u}_{H}\right\|_{L^{2}\left(\Omega_{i}\right)}^{2}\right) \\
& \leq C k_{i, M} C_{H, h}^{2}\left\|\mathcal{E}_{i} \boldsymbol{w}^{(i)}\right\|_{L^{2}\left(\Omega_{i}\right)}^{2} \\
& \leq C \frac{k_{i, M}}{k_{i, m}} C_{H, h}^{2} \mathbf{a}_{i}\left(\mathcal{E}_{i} \boldsymbol{w}^{(i)}, \mathcal{E}_{i} \boldsymbol{w}^{(i)}\right) \\
& =C \frac{k_{i, M}}{k_{i, m}} C_{H, h}^{2}\left\|\boldsymbol{w}^{(i)}\right\|_{S_{\Gamma}^{(i)}}^{2} .
\end{aligned}
$$

Lemma 4.8. For a benign subdomain $\Omega_{i}$, we have

$$
\left\|R_{F}^{T}\left(\boldsymbol{w}_{F}^{(i)}-\boldsymbol{w}_{\Pi_{F}}\right)\right\|_{S_{\Gamma}^{(i)}}^{2} \leq C \frac{k_{i, M}}{k_{i, m}} C_{H, h}^{2}\left\|\boldsymbol{w}^{(i)}\right\|_{S_{\Gamma}^{(i)}}^{2},
$$

where $C$ is independent of $N, h, H$, and the coefficients of $K_{p}$ and $w_{i}$.

Proof. We have

$$
\left\|R_{F}^{T}\left(\boldsymbol{w}_{F}^{(i)}-\boldsymbol{w}_{\Pi_{F}}\right)\right\|_{S_{\Gamma}^{(i)}}^{2}=\mathbf{a}_{i}\left(\boldsymbol{r}_{u}, \boldsymbol{r}_{u}\right)+\mathbf{c}_{i}\left(r_{p}, r_{p}\right),
$$

where $\left(\boldsymbol{r}_{u}, r_{p}\right) \in \boldsymbol{W}^{(i)} \times Q^{(i)}$ is the discrete Darcy extension to the subdomain interior given the boundary value $\zeta_{F}\left(\boldsymbol{w}^{(i)}-\boldsymbol{w}_{\Pi_{F}}\right) \cdot \boldsymbol{n}$. Let $\left(\boldsymbol{w}_{u}, w_{p}\right) \in \boldsymbol{W}^{(i)} \times Q^{(i)}$ be the discrete Darcy extension of $\boldsymbol{w}^{(i)}$ given $\boldsymbol{w}^{(i)} \cdot \boldsymbol{n}$ on $\partial \Omega_{i}$. We have

$$
\left\|\boldsymbol{w}^{(i)}\right\|_{S_{\Gamma}^{(i)}}^{2}=\mathbf{a}_{i}\left(\boldsymbol{w}_{u}, \boldsymbol{w}_{u}\right)+\mathbf{c}_{i}\left(w_{p}, w_{p}\right)
$$

and $\mathbf{b}_{i}\left(\boldsymbol{w}_{u}, q\right)-\mathbf{c}_{i}\left(w_{p}, q\right)=0 \forall q \in Q^{(i)} \cdot q=\nabla \cdot \boldsymbol{w}_{u}$, we have

$$
\left\|\nabla \cdot \boldsymbol{w}_{u}\right\|_{L^{2}\left(\Omega_{i}\right)}^{2} \leq w_{i} \mathbf{c}_{i}\left(w_{p}, w_{p}\right) .
$$

By [41, Lemma 4.8], given two positive constants $\alpha$ and $\beta$, there exist $\boldsymbol{v}_{i, F} \in \boldsymbol{W}^{(i)}$ and $\boldsymbol{v}_{i, F}^{H} \in \boldsymbol{W}^{H}$ such that

$$
\left\{\begin{array} { l l } 
{ \boldsymbol { v } _ { i , F } = \boldsymbol { w } _ { u } } & { \text { in } F , } \\
{ 0 , } & { \partial \Omega _ { i } \backslash F , }
\end{array} \quad \left\{\begin{array}{ll}
\boldsymbol{v}_{i, F}^{H}=\Pi_{R T}^{H} \boldsymbol{w}_{u} & \text { in } F, \\
0, & \partial \Omega_{i} \backslash F,
\end{array}\right.\right.
$$

Copyright $@$ ㅇ by SIAM. Unauthorized reproduction of this article is prohibited. 
and

$\alpha\left\|\nabla \cdot\left(\boldsymbol{v}_{i, F}-\boldsymbol{v}_{i, F}^{H}\right)\right\|_{L^{2}\left(\Omega_{i}\right)}^{2}+\beta\left\|\boldsymbol{v}_{i, F}-\boldsymbol{v}_{i, F}^{H}\right\|_{L^{2}\left(\Omega_{i}\right)}^{2} \leq C C_{H, h}^{2}\left(\alpha\left\|\nabla \cdot \boldsymbol{w}_{u}\right\|_{L^{2}\left(\Omega_{i}\right)}^{2}+\beta\left\|\boldsymbol{w}_{u}\right\|_{L^{2}\left(\Omega_{i}\right)}^{2}\right)$,

where $C$ is independent of $\alpha, \beta, h$, and $H$.

Let $p_{i, F}$ satisfy $\mathbf{b}\left(\boldsymbol{v}_{i, F}-\boldsymbol{v}_{i, F}^{H}, q\right)-\mathbf{c}\left(p_{i, F}, q\right)=0 \forall q \in Q^{(i)}$. Taking $q=p_{i, F}$,

$$
\mathbf{c}_{i}\left(p_{i, F}, p_{i, F}\right) \leq \frac{1}{w_{i}}\left\|\nabla \cdot\left(\boldsymbol{v}_{i, F}-\boldsymbol{v}_{i, F}^{H}\right)\right\|_{L^{2}\left(\Omega_{i}\right)}^{2} .
$$

Since $\left(\boldsymbol{v}_{i, F}-\boldsymbol{v}_{i, F}^{H}\right) \cdot \boldsymbol{n}=\zeta_{F}\left(\boldsymbol{w}^{(i)}-\boldsymbol{w}_{\Pi_{F}}\right) \cdot \boldsymbol{n}$ on $\partial \Omega_{i}$, using the energy-minimizing property of the discrete Darcy extension, (53), (52), (51), and (50), we can bound (49) by

$$
\begin{aligned}
& \mathbf{a}_{i}\left(\boldsymbol{r}_{u}, \boldsymbol{r}_{u}\right)+\mathbf{c}_{i}\left(r_{p}, r_{p}\right) \leq \mathbf{a}_{i}\left(\boldsymbol{v}_{i, F}-\boldsymbol{v}_{i, F}^{H}, \boldsymbol{v}_{i, F}-\boldsymbol{v}_{i, F}^{H}\right)+\mathbf{c}_{i}\left(p_{i, F}, p_{i, F}\right) \\
& \leq k_{i, M}\left\|\boldsymbol{v}_{i, F}-\boldsymbol{v}_{i, F}^{H}\right\|_{L^{2}\left(\Omega_{i}\right)}^{2}+\frac{1}{w_{i}}\left\|\nabla \cdot\left(\boldsymbol{v}_{i, F}-\boldsymbol{v}_{i, F}^{H}\right)\right\|_{L^{2}\left(\Omega_{i}\right)}^{2} \\
& \leq C C_{H, h}^{2}\left(k_{i, M}\left\|\boldsymbol{w}_{u}\right\|_{L^{2}\left(\Omega_{i}\right)}^{2}+\frac{1}{w_{i}}\left\|\nabla \cdot \boldsymbol{w}_{u}\right\|_{L^{2}\left(\Omega_{i}\right)}^{2}\right) \\
& \leq C C_{H, h}^{2}\left(k_{i, M}\left\|\boldsymbol{w}_{u}\right\|_{L^{2}\left(\Omega_{i}\right)}^{2}+\frac{1}{w_{i}} w_{i} \mathbf{c}_{i}\left(w_{p}, w_{p}\right)\right) \\
& \leq C C_{H, h}^{2} \frac{k_{i, M}}{k_{i, m}}\left(\mathbf{a}_{i}\left(\boldsymbol{w}_{u}, \boldsymbol{w}_{u}\right)+\mathbf{c}_{i}\left(w_{p}, w_{p}\right)\right) \\
& =C \frac{k_{i, M}}{k_{i, m}} C_{H, h}^{2}\left\|\boldsymbol{w}^{(i)}\right\|_{S_{\Gamma}^{(i)}}^{2} .
\end{aligned}
$$

We are ready to state our main result with the assumptions made at the beginning of this section.

THEOREM 4.9 (minimal coarse space). Let the assumptions of Theorem 4.1 hold. Then, the preconditioned BDDC operator for (4) will satisfy

$$
\kappa\left(M^{-1} K\right)=\kappa\left(M_{\Gamma}^{-1} \widehat{S}\right) \leq C \max _{1 \leq i \leq N} \frac{k_{i, M}}{k_{i, m}} C_{H, h}^{2},
$$

where $C$ is independent of $N, h, H$, and the coefficients of $K_{p}$ and $w$.

Proof. Equation (38) provides an upper bound of the square of the $S_{\Gamma}^{(i)}$-norm of a face component of the average operator; it can be further bounded using the face lemmas Lemmas 4.7 and 4.8 for malign and benign subdomains, respectively. Using Theorem 4.1, we obtain our result.

5. Numerical experiments. The triangulation of $\Omega$ and the assembly of the subdomain matrices is performed with the DOLFIN library [33], which is part of the FEniCS project [32]. ParMETIS [22] is used to decompose the meshes, and each subdomain is assigned to a different MPI process. MUMPS [1] is used for the subdomain interior solvers and for the explicit computation of local Schur complements. Additional details on the implementation can be found in [63]. A relative residual reduction of $10^{-8}$ is used as the stopping criterion of the conjugate gradient method; random right-hand sides and zero initial guesses are always considered. Unless otherwise stated, the domain is $\Omega=[0,1]^{3}, K_{p}=I_{3}, w=0$, and the primal space considered is the minimal one which guarantees the no-net-flux condition (14).

Copyright (c) by SIAM. Unauthorized reproduction of this article is prohibited. 

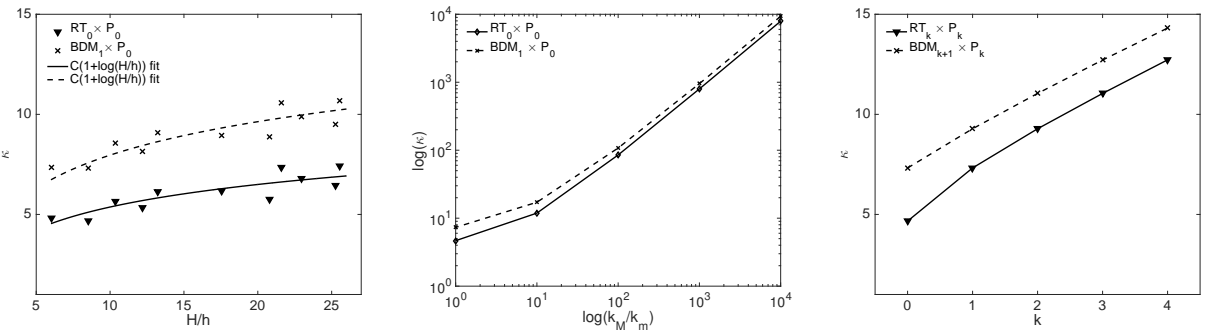

FIG. 1. Left: Quasi-optimality test. $\kappa$ as a function of $H / h$. Center: $\kappa$ as a function of $k_{M} / k_{m}$. Right: Polynomial order dependence. $\kappa$ as a function of the order $(H / h=8.5)$. Results for $R T_{0} \times P_{0}$ (triangles) and $B D M_{1} \times P_{0}$ (crosses).

Large scale numerical results have been obtained on the Cray XC40 Shaheen of KAUST, ranked 10th in the Top500 list as of June 2016, and which features 6192 nodes, each of them equipped with dual 16-core Haswell processors and 128 GB of DRAM.

5.1. Quasi-optimality. We consider decompositions of $\Omega$ into 40 subdomains obtained from a sequence of meshes with decreasing $h . K_{p}=c I_{3}$, where $c$ is set to 1000 in the subdomains identified by even MPI ranks and to 0.01 otherwise. Experimental condition numbers are shown in the left panel of Figure 1 as a function of $H / h:=$ $\max _{i}\left\{H_{i} / h_{i}\right\}$, where $H_{i}=\left\{\max _{P_{1}, P_{2} \in \partial \Omega_{i, h}} d\left(P_{1}, P_{2}\right)\right\}$ with $P_{1}$ and $P_{2}$ two vertices of the boundary mesh $\partial \Omega_{i, h}$, and $d\left(P_{1}, P_{2}\right)$ their Euclidean distance.

The numerical results confirm Theorem 4.9 for the $\mathrm{RT}_{0} \times \mathrm{P}_{0}$ case and validate the method for $\mathrm{BDM}_{1} \times \mathrm{P}_{0}$, since the experimental condition numbers increase slightly as $H / h$ increases and the results are independent of the jumps in $K_{p}$. A fitting of the data indicates that $\kappa$ grows as $C_{H, h}$, and not as $C_{H, h}^{2}$. Similar results have been obtained using exponentially random (element by element) distributed $w$ (data not shown). In the central panel of Figure 1, we report on the condition number dependence of $k_{M} / k_{m}$, by considering a fixed mesh decomposed into 40 subdomains ( $h=0.1, H / h=8.5$ ), and by using $K_{p}=\operatorname{diag}\left\{k_{1}, 1,1\right\}$ with $k_{1}$ going from 1 to 10000; the numerical results confirm that our estimates are sharp.

5.2. Dependence on the polynomial order. Although our theory does not cover the case of BDM elements or RT elements of higher order, in Figure 1 (right panel) we report on the experimental condition numbers obtained by considering a fixed mesh $(h=0.1, H / h=8.5)$, and by increasing the polynomial order $(k)$ of the FE pairs. A logarithmic dependence of the condition number can be conjectured.

5.3. Adaptive primal spaces. In Figure 2, we present results using our adaptive algorithm. We fix the mesh and the domain decomposition $(h=0.1, H / h=8.5)$, and we investigate the quality of the adaptive coarse spaces generated by using deluxe (diamond marks) or cardinality (crosses) scaling, testing for RT (continuous lines) and BDM (dashed) velocity spaces. In the left column, we plot the experimental condition number as a function of the prescribed tolerance $\nu_{t o l}$ for the eigenvalue problems (40) and (42); the central and right columns provide additional insights into the efficiency of these eigenvalue problems. The ratio between the number of primal and interface dofs (coarse ratio) is shown as a function of $\nu_{t o l}$ in the central column; the right column shows the number of iterations as a function of the coarse ratio.

In a first set of tests (Figure 2, top row), we consider $K_{p}=I_{3} ; \nu_{t o l}$ is shown to be a very good indicator of $\kappa$ with both types of scalings and FE pairs. The adap- 

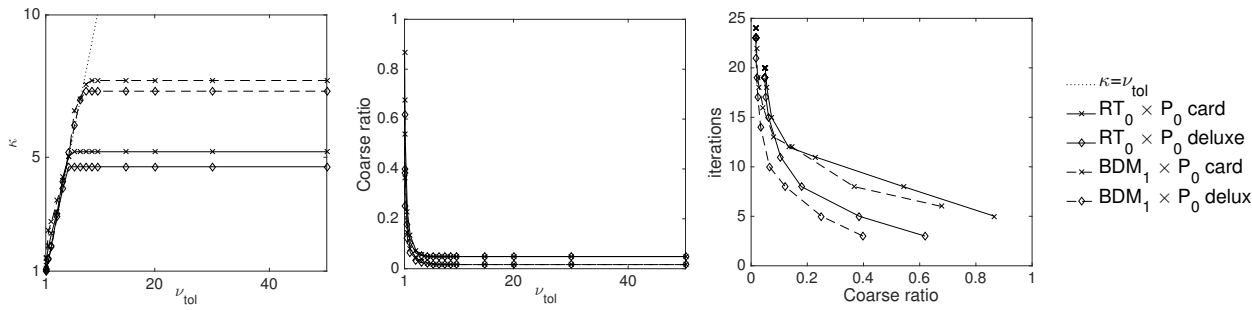

$-x \mathrm{BDM}_{1} \times \mathrm{P}_{0}$ card

$\rightarrow \mathrm{BDM}_{1} \times \mathrm{P}_{0}^{0}$ deluxe
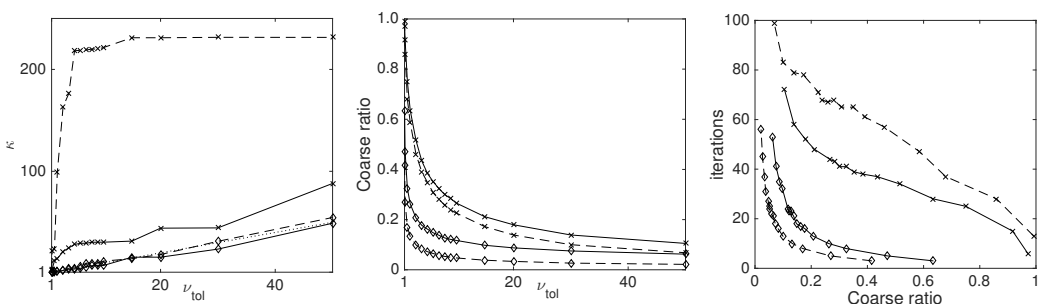

$\ldots . . . \kappa=\nu_{\text {tol }}$
$*-\mathrm{RT}_{0} \times \mathrm{P}_{0}$ card

$\rightarrow$ RT $T_{0} \times \mathrm{P}_{0}$ deluxe

$\rightarrow \mathrm{BDM}_{1} \times \mathrm{P}_{0}$ card $\rightarrow \mathrm{BDM}_{1} \times \mathrm{P}_{0}$ deluxe
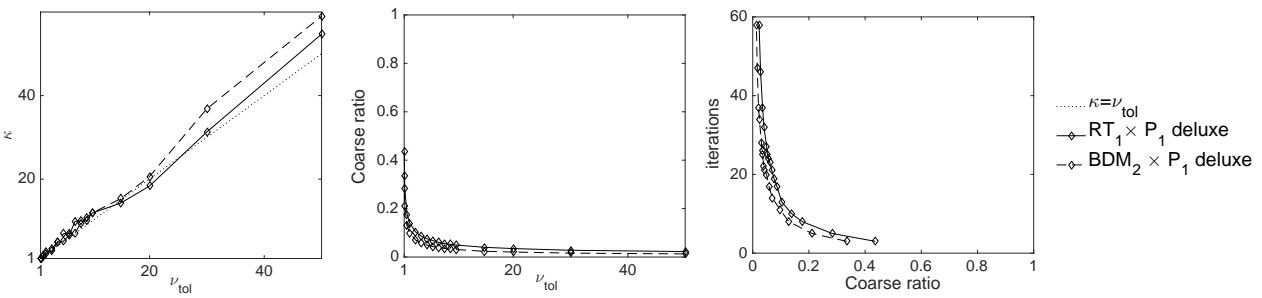

FIG. 2. Adaptive BDDC with different scaling. Deluxe (diamond marks) compared against cardinality (crosses). Top row: $K_{p}=I_{3}$. Central and bottom row $K_{p}=\operatorname{diag}\left\{k_{1}, k_{2}, k_{3}\right\}, k_{i}$ elementwise exponentially random in $\left[10^{-2}, 10^{2}\right]$. Left: $\kappa$ versus $\nu_{\text {tol }}$. Center: coarse ratio versus $\nu_{\text {tol }}$. Right: iterations versus coarse ratio.

tive technique proved to be very efficient; no primal functions, in addition to those needed for enforcing the no-net-flux condition, are selected when using large values of $\nu_{t o l}$. For the results provided in the middle row, we instead consider a randomly distributed $K_{p}=\operatorname{diag}\left\{k_{1}, k_{2}, k_{3}\right\}$ with $k_{i}$ exponentially distributed in $\left[10^{-2}, 10^{2}\right]$. Adaptive BDDC deluxe generates efficient and robust primal spaces, as the resulting $\kappa$ is very close to $\nu_{t o l}$; instead, cardinality scaling leads to worse approximation properties, especially with the pair $\mathrm{BDM}_{1} \times \mathrm{P}_{0}$. The size of the generated primal space in the deluxe case is always smaller than that generated with the cardinality scaling, in alignment with observations in [23, 24] and theory [45]. In the last row, we report the results using adaptive BDDC deluxe with higher order spaces and randomly distributed $K_{p}$; even in this case, the adaptive technique results are efficient and robust. Noticeably, a more favorable coarsening is obtained using $\mathrm{BDM}_{2} \times \mathrm{P}_{1}$. Overall, the results indicate that the constant $C$ appearing in Theorem 4.3 is not as unpleasant as the theory predicts.

5.4. Multilevel results. In this section, we report numerical results to validate our multilevel algorithm. For this test, we triangulate the unit cube and decompose the mesh into 2048 subdomains; we set $K_{p}=I_{3}$. We consider the finite element pair $\mathrm{RT}_{0} \times \mathrm{P}_{0}$; the total number of dofs is $46 \mathrm{M}$, split into $31 \mathrm{M}$ for the velocity and $15 \mathrm{M}$ for the pressure field. Natural boundary conditions are considered; this implies that the subdomains that touch $\partial \Omega$ are benign. Coarser subdomains are obtained by aggregating 16 subdomains at the finer level. The number of subdomains at level 1 is 
TABLE 1

Multilevel BDDC. $\kappa$ and number of iterations for different multilevel preconditioners $M_{m l, 2}^{-1}$, $M_{m l, 3}^{-1}, M_{m l, 4}^{-1}$, and coarser $B D D C M_{1}^{-1}$ and $M_{2}^{-1}$.

\begin{tabular}{ccccc}
\hline$M_{m l, 2}^{-1}$ & $M_{m l, 3}^{-1}$ & $M_{m l, 4}^{-1}$ & $M_{1}^{-1}$ & $M_{2}^{-1}$ \\
\hline \hline $12.9 / 30$ & $20.2 / 35$ & $25.9 / 36$ & $4.67 / 12$ & $2.09 / 6$ \\
\hline
\end{tabular}
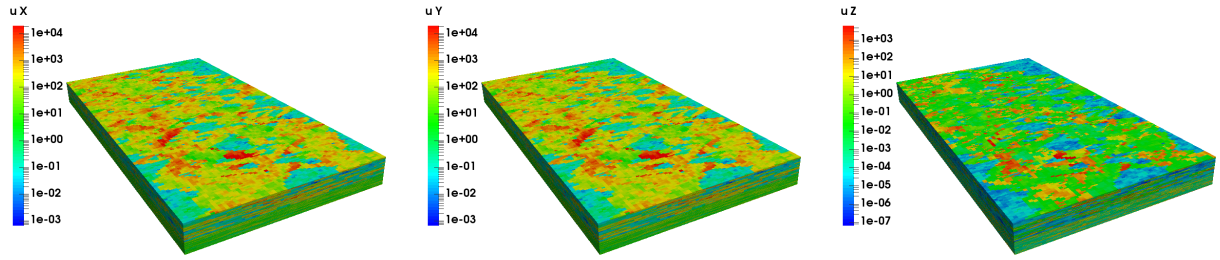

FIG. 3. $X-, \quad Y$-, and Z-components of the permeability tensor from the second dataset of the SPE10 benchmark [49]. Values are shown in logarithmic scale.

128 , and the number of dofs is $12 \mathrm{~K}$; at level 2 , the subdomains are 8 and the number of dofs is $1 \mathrm{~K}$. The coarse spaces, at each level of the hierarchy, are the minimal ones that guarantee the no-net-flux condition.

In Table 1 we report the condition number and the number of iterations for the multilevel preconditioners $M_{m l, 2}^{-1}$ (standard two-level BDDC), $M_{m l, 3}^{-1}$ (three levels), and $M_{m l, 4}^{-1}$ (four levels), together with the condition number of the coarser BDDC operators $M_{1}^{-1}$ and $M_{2}^{-1}$. Note that $M_{1}^{-1}$ has been computed by using an exact coarse solver. The results confirm the multilevel theory presented in section 3.5 and validate our implementation.

5.5. SPE10 benchmark. We finally report on using adaptive multilevel BDDC with a real-world, high-contrast distribution of permeabilities. The domain considered is $1200 \mathrm{ft} \times 2200 \mathrm{ft} \times 170 \mathrm{ft}$, regularly decomposed in a hexahedral grid $60 \times 220 \times 85$ as given by the second dataset of the SPE10 benchmark [49]. Since DOLFIN does not currently support hexahedral elements, each hexahedron is further subdivided into 6 tetrahedra with high aspect ratios. The total number of cells is approximatively $6.7 \mathrm{M}$; we consider the pairs $\mathrm{RT}_{0} \times \mathrm{P}_{0}$ and $\mathrm{BDM}_{1} \times \mathrm{P}_{0}$ which lead to approximatively $20 \mathrm{M}$ and $47 \mathrm{M}$ dofs, respectively. The permeability tensor from the SPE10 benchmark is of the form $K_{p}=\operatorname{diag}\left\{k_{1}, k_{2}, k_{3}\right\}$; the values for $k_{i}$ are pictured in Figure 3. Preliminary results have been already published in [65].

The results in the top row of Table 2 (left panel RT, right panel BDM) confirm that adaptive BDDC deluxe can control the condition number and the number of iterations with many subdomains and highly heterogeneous, real-world coefficient distributions. We also report on the coarsening ratios of the adaptive procedure, here expressed either as the ratio between fine and coarse dofs, or between interface and coarse dofs. As it is a strong scaling setting, the coarsening gets worse as the number of subdomains is increased; however, with $1 \mathrm{~K}$ subdomains, the adaptive technique is able to coarsen with high ratios, with larger ratios obtained with the pair $\mathrm{BDM}_{1} \times \mathrm{P}_{0}$.

We finally report the timings associated with the setup of the preconditioner and the Krylov solver from the previous set of results (see Table 3, top row), and we compare them to three-level adaptive BDDC deluxe methods (central row); reported are also the condition number and the number of iterations for the three-level methods 
TABLE 2

Adaptive BDDC deluxe: SPE10 benchmark. Top: $\kappa$ and number of iterations as a function of $\nu_{\text {tol }}$ and the number of subdomains N. Bottom: coarsening ratios.

\begin{tabular}{|c|c|c|c|c|c|c|c|c|c|}
\hline \multicolumn{7}{|c|}{$\mathrm{RT}_{0} \times \mathrm{P}_{0}$} & \multicolumn{3}{|c|}{$\mathrm{BDM}_{1} \times \mathrm{P}_{0}$} \\
\hline \multicolumn{10}{|c|}{$\kappa /$ Iterations } \\
\hline$N$ & $\nu_{t o l}=10$ & $\nu_{t o l}=5$ & $\nu_{t o l}=2.5$ & $\nu_{t o l}=1.5$ & $N$ & $\nu_{t o l}=10$ & $\nu_{t o l}=5$ & $\nu_{t o l}=2.5$ & $\nu_{t o l}=1.5$ \\
\hline 1024 & $15.9 / 25$ & $7.77 / 17$ & $3.57 / 11$ & $1.73 / 6$ & 1024 & $16.1 / 24$ & $7.51 / 16$ & $3.49 / 10$ & $1.60 / 6$ \\
\hline 2048 & $15.0 / 25$ & $7.76 / 17$ & $3.51 / 11$ & $1.65 / 6$ & 2048 & $16.7 / 25$ & $7.45 / 16$ & $3.53 / 10$ & $1.61 / 6$ \\
\hline 4096 & $15.4 / 25$ & $8.19 / 18$ & $3.42 / 11$ & $1.68 / 6$ & 4096 & $15.5 / 24$ & $7.53 / 16$ & $3.57 / 10$ & $1.58 / 6$ \\
\hline 8192 & $16.5 / 26$ & $7.69 / 17$ & $3.51 / 11$ & $1.67 / 6$ & 8192 & $15.9 / 24$ & $7.77 / 17$ & $3.53 / 10$ & $1.59 / 6$ \\
\hline
\end{tabular}

Fine to coarse ratio / Interface to coarse ratio

\begin{tabular}{|c|c|c|c|c|c|c|c|c|c|}
\hline$N$ & $\nu_{t o l}=10$ & $\nu_{t o l}=5$ & $\nu_{t o l}=2.5$ & $\nu_{t o l}=1.5$ & $N$ & $\nu_{t o l}=10$ & $\nu_{t o l}=5$ & $\nu_{t o l}=2.5$ & $\nu_{t o l}=1.5$ \\
\hline 1024 & $745 / 25$ & $481 / 16$ & $281 / 9$ & $172 / 5$ & 1024 & $1378 / 61$ & $795 / 35$ & $408 / 18$ & $230 / 10$ \\
\hline 2048 & $402 / 17$ & $272 / 11$ & $165 / 7$ & $105 / 4$ & 2048 & $761 / 42$ & $458 / 25$ & $243 / 13$ & $140 / 7$ \\
\hline 4096 & $218 / 11$ & $153 / 8$ & $98 / 5$ & $64 / 3$ & 4096 & $420 / 29$ & $263 / 18$ & $146 / 10$ & $86 / 6$ \\
\hline 8192 & $121 / 8$ & $89 / 5$ & $60 / 4$ & $41 / 2$ & 8192 & $236 / 20$ & $155 / 13$ & $90 / 7$ & $54 / 4$ \\
\hline
\end{tabular}

TABLE 3

Adaptive BDDC deluxe: SPE10 benchmark. Setup/solving times (in seconds) for two-level $B D D C$ (top) and three-level BDDC (center) as a function of $\nu_{\text {tol }}$ and the number of subdomains $N$. Bottom: condition number and number of iterations for three-level BDDC.

\begin{tabular}{|c|c|c|c|c|c|c|c|c|c|}
\hline \multicolumn{5}{|c|}{$\mathrm{RT}_{0} \times \mathrm{P}_{0}$} & \multicolumn{5}{|c|}{$\mathrm{BDM}_{1} \times \mathrm{P}_{0}$} \\
\hline & & & Two-l & BDDC & etup & Solve tir & nes & & \\
\hline$N$ & $\nu_{t o l}=10$ & $\nu_{t o l}=5$ & $\nu_{t o l}=2.5$ & $\nu_{t o l}=1.5$ & $N$ & $\nu_{t o l}=10$ & $\nu_{t o l}=5$ & $\nu_{t o l}=2.5$ & $\nu_{t o l}=1.5$ \\
\hline 1024 & $3.4 / 1.1$ & $3.9 / 0.9$ & $5.3 / 0.8$ & $8.7 / 0.9$ & 1024 & $25.6 / 4.7$ & $26.1 / 3.4$ & $31.0 / 2.9$ & $47.7 / 3.1$ \\
\hline 2048 & $3.3 / 1.1$ & $4.4 / 1.1$ & $6.0 / 1.2$ & $11.1 / 1.2$ & 2048 & $10.6 / 2.4$ & $12.0 / 1.9$ & $18.2 / 2.1$ & $61.5 / 3.6$ \\
\hline 4096 & $5.6 / 1.7$ & $7.4 / 1.7$ & $11.0 / 1.5$ & $20.8 / 2.1$ & 4096 & $7.9 / 2.4$ & $9.8 / 2.2$ & $27.4 / 3.0$ & $130.9 / 4.3$ \\
\hline 8192 & $9.6 / 3.0$ & $15.8 / 4.4$ & $21.0 / 3.6$ & $48.1 / 3.9$ & 8192 & $15.7 / 3.5$ & $18.2 / 5.8$ & $50.2 / 5.9$ & $390.5 / 5.4$ \\
\hline
\end{tabular}

Three-level BDDC: Setup / Solve times

\begin{tabular}{ccccccrrrrr}
$N$ & $\nu_{t o l}=10$ & $\nu_{t o l}=5$ & $\nu_{t o l}=2.5$ & $\nu_{\text {tol }}=1.5$ & & $N$ & $\nu_{t o l}=10$ & $\nu_{t o l}=5$ & $\nu_{t o l}=2.5$ & $\nu_{t o l}=1.5$ \\
\hline \hline 1024 & $3.7 / 1.4$ & $6.4 / 1.2$ & $6.5 / 1.2$ & $6.6 / 1.6$ & & 1024 & $24.2 / 5.7$ & $25.2 / 3.9$ & $28.3 / 4.4$ & $41.7 / 5.3$ \\
2048 & $2.9 / 1.1$ & $3.9 / 1.2$ & $4.4 / 1.1$ & $5.5 / 1.5$ & & 2048 & $10.2 / 2.8$ & $10.7 / 2.2$ & $14.7 / 2.9$ & $20.2 / 3.6$ \\
4096 & $4.9 / 1.2$ & $5.3 / 1.4$ & $5.8 / 1.3$ & $6.0 / 1.2$ & & 4096 & $6.2 / 2.1$ & $7.2 / 1.8$ & $12.3 / 2.9$ & $11.5 / 2.6$ \\
8192 & $7.8 / 2.0$ & $7.8 / 1.6$ & $7.9 / 2.1$ & $9.1 / 1.8$ & & 8192 & $9.2 / 2.4$ & $11.7 / 2.5$ & $11.9 / 2.1$ & $13.9 / 2.3$ \\
\hline
\end{tabular}

Three-level BDDC: $\kappa /$ Iterations

\begin{tabular}{ccccccccccc}
$N$ & $\nu_{\text {tol }}=10$ & $\nu_{\text {tol }}=5$ & $\nu_{\text {tol }}=2.5$ & $\nu_{\text {tol }}=1.5$ & & $N$ & $\nu_{\text {tol }}=10$ & $\nu_{\text {tol }}=5$ & $\nu_{\text {tol }}=2.5$ & $\nu_{\text {tol }}=1.5$ \\
\hline \hline 1024 & $22.4 / 30$ & $11.0 / 21$ & $5.4 / 14$ & $2.7 / 9$ & & 1024 & $24.7 / 29$ & $12.0 / 21$ & $5.7 / 14$ & $2.5 / 8$ \\
2048 & $21.6 / 30$ & $12.0 / 22$ & $5.9 / 15$ & $2.8 / 9$ & & 2048 & $23.7 / 29$ & $11.8 / 20$ & $5.6 / 14$ & $2.6 / 9$ \\
4096 & $24.0 / 32$ & $12.3 / 22$ & $5.4 / 14$ & $2.6 / 9$ & & 4096 & $24.9 / 31$ & $12.5 / 21$ & $5.6 / 14$ & $2.5 / 9$ \\
8192 & $27.1 / 34$ & $11.9 / 22$ & $5.5 / 14$ & $2.7 / 9$ & & 8192 & $24.0 / 30$ & $12.7 / 22$ & $5.9 / 14$ & $2.6 / 9$ \\
\hline
\end{tabular}

(bottom row). For the three-level case, 16 subdomains are aggregated into a coarse subdomain, and the coarse space for the second level is adaptively generated using an eigenvalue threshold of 10 . The application of the coarse problem at the finest level is accelerated using 2 iterations of the Chebyshev iterative method (using $[1,10]$ as the approximation of the preconditioned spectrum). On the other hand, the coarse problem for the two-level method is solved in parallel on a subcommunicator, $1 \mathrm{~K}$ coarse equations per coarse MPI process.

The optimal granularity (with one MPI process per subdomain) is at $1 \mathrm{~K}$ cores for $\mathrm{RT}_{0} \times \mathrm{P}_{0}$, while it is at $4 \mathrm{~K}$ cores for the $\mathrm{BDM}_{1} \times \mathrm{P}_{0}$, since the number of local dofs in the latter case is higher. The Krylov solving times decrease for smaller values of $\nu_{t o l}$, at a cost of an increase in setup times, which become dramatic when approaching the 
condition number limit, since the factorization step of the coarse problem tends to dominate the computations. Multilevel adaptive BDDC deluxe methods are shown to be robust and faster alternatives than their two-level counterparts, since they are able to provide high-quality preconditioning even when using small values for $\nu_{t o l}$ and a larger number of subdomains. The optimal tuning of $\nu_{t o l}$ and the other parameters of the multilevel method are clearly application dependent, and are strongly influenced by the possibility of reusing the preconditioner for multiple right-hand sides.

Acknowledgments. The authors would like to thank the two anonymous referees for their comments and suggestions that helped improve the quality of the manuscript. For computer time, this research used the resources of the Supercomputing Laboratory at King Abdullah University of Science \& Technology (KAUST) in Thuwal, Saudi Arabia.

\section{REFERENCES}

[1] P. R. Amestoy, I. S. Duff, J.-Y. L'Excellent, And J. Koster, A fully asynchronous multifrontal solver using distributed dynamic scheduling, SIAM J. Matrix Anal. Appl., 23 (2001), pp. $15-41$.

[2] W. N. Anderson, JR., And R. J. Duffin, Series and parallel addition of matrices, J. Math. Anal. Appl., 26 (1969), pp. 576-594.

[3] D. N. Arnold, D. Boffi, and R. S. Falk, Quadrilateral H(div) finite elements, SIAM J. Numer. Anal., 42 (2005), pp. 2429-2451.

[4] S. Balay, S. Abhyankar, M. F. Adams, J. Brown, P. Brune, K. Buschelman, L. Dalcin, V. Eijkhout, W. D. Gropp, D. Kaushik, M. G. Knepley, L. C. McInnes, K. Rupp, B. F. Smith, S. Zampini, And H. Zhang, PETSc Users Manual, Technical report ANL95/11-Revision 3.6, Argonne National Laboratory, Argonne, IL, 2015.

[5] L. Beirão da Veiga, L. F. Pavarino, S. Scacchi, O. B. Widlund, and S. Zampini, Isogeometric BDDC preconditioners with deluxe scaling, SIAM J. Sci. Comput., 36 (2014), pp. A1118-A1139.

[6] L. Beirão da Veiga, L. F. Pavarino, S. Scacchi, O. B. Widlund, and S. Zampini, Adaptive selection of primal constraints for isogeometric BDDC deluxe preconditioners, SIAM J. Sci. Comput., 39 (2017), pp. A281-A302.

[7] D. Boffi, F. BreAzzi, And M. Fortin, Mixed Finite Element Methods and Applications, Springer, Heidelberg, 2013.

[8] J. G. Calvo and O. B. Widlund, An Adaptive Choice of Primal Constraints for BDDC Domain Decomposition Algorithms, Technical report TR2015-979, Courant Institute of Mathematical Sciences, New York, 2015.

[9] Z. Chen, G. Hunn, And Y. Ma, Computational Methods for Multiphase Flows in Porous Media, Comput. Sci. Eng., SIAM, Philadelphia, 2006.

[10] C. R. Dohrman, A Substructuring Preconditioner for Nearly Incompressible Elasticity Problems, Technical report, SAND 2004-5393, Sandia National Laboratories, Albuquerque, New Mexico, 2004.

[11] C. R. Dohrmann, A preconditioner for substructuring based on constrained energy minimization, SIAM J. Sci. Comput., 25 (2003), pp. 246-258.

[12] C. R. Dohrmann, An approximate BDDC preconditioner, Numer. Linear Algebra Appl., 14 (2007), pp. 149-168.

[13] C. R. Dohrmann and P. B. Bochev, A stabilized finite element method for the Stokes problem based on polynomial pressure projections, Internat. J. Numer. Methods Fluids, 46 (2004), pp. 183-201.

[14] C. R. Dohrmann And O. B. Widlund, A BDDC algorithm with deluxe scaling for threedimensional H(curl) problems, Comm. Pure Appl. Math., 69 (2016), pp. 745-770.

[15] R. E. EwIng AND J. WANG, Analysis of the Schwarz algorithm for mixed finite element methods, ESAIM Math. Model. Numer. Anal., 26 (1992), pp. 739-756.

[16] R. S. Falk, P. Gatto, And P. Monk, Hexahedral H(div) and H(curl) finite elements, ESAIM Math. Model. Numer. Anal., 45 (2011), pp. 115-143.

[17] C. Farhat, M. Lesoinne, P. LeTallec, K. Pierson, and D. Rixen, FETI-DP: A dual-primal unified FETI method. I. A faster alternative to the two-level FETI method, Internat. J. Numer. Methods Engrg., 50 (2001), pp. 1523-1544. 
[18] V. Girault and P.-A. Raviart, Finite Element Methods for Navier-Stokes Equations. Springer, New York, 1986.

[19] P. Goldfeld, Balancing Neumann-Neumann Preconditioners for the Mixed Formulation of Almost-Incompressible Linear Elasticity. Ph.D. thesis, New York University, Courant Institute of Mathematical Sciences, New York, 2003.

[20] P. HÉnon, P. RAmet, And J. Roman, PASTIX: A high-performance parallel direct solver for sparse symmetric positive definite systems, Parallel Comput., 28 (2002), pp. 301-321.

[21] R. Ingram, M. F. Wheeler, and I. Yotov, A multipoint flux mixed finite element method on hexahedra, SIAM J. Numer. Anal., 48 (2010), pp. 1281-1312.

[22] G. Karypis, METIS and ParMETIS, in Encyclopedia of Parallel Computing, D. Padua, ed., Springer, New York, 2011, pp. 1117-1124.

[23] H. H. Kim, E. T. Chung, AND J. WANG, BDDC and FETI-DP Algorithms with Adaptive Coarse Spaces for Three-Dimensional Elliptic Problems with Oscillatory and High Contrast Coefficients, preprint, arXiv:1606.07560, 2016.

[24] A. Klawonn, M. Kühn, And O. Rheinbach, Adaptive coarse spaces for FETI-DP in three dimensions, SIAM J. Sci. Comput., 38 (2016), pp. A2880-A2911.

[25] A. Klawonn, P. Radtke, And O. Rheinbach, A comparison of adaptive coarse spaces for iterative substructuring in two dimensions, Electron. Trans. Numer. Anal., 45 (2016), pp. 75106.

[26] A. Klawonn and O. B. Widlund, Dual-primal FETI methods for linear elasticity, Comm. Pure Appl. Math., 59 (2006), pp. 1523-1572.

[27] A. Kuzmin, M. Luisier, And O. Schenk, Fast methods for computing selected elements of the greens function in massively parallel nanoelectronic device simulations, in Euro-Par 2013 Parallel Processing, F. Wolf, B. Mohr, and D. Mey, ed., Lecture Notes in Comput. Sci. 8097, Springer, Berlin, 2013, pp. 533-544.

[28] M. la Cour Christensen, U. Villa, A. P. Engsig-Karup, and P. S. Vassilevski, Numerical multilevel upscaling for incompressible flow in reservoir simulation: An element-based algebraic multigrid (AMGe) approach, SIAM J. Sci. Comput., 39 (2017), pp. B102-B137.

[29] J. Li ANd X. TU, A nonoverlapping domain decomposition method for incompressible Stokes equations with continuous pressures, SIAM J. Numer. Anal., 51 (2013), pp. 1235-1253.

[30] J. Li AND O. B. Widlund, BDDC algorithms for incompressible Stokes equations, SIAM J. Numer. Anal., 44 (2006), pp. 2432-2455.

[31] J. Li And O. B. WidLund, FETI-DP, BDDC, and block Cholesky methods, Internat. J. Numer. Methods Engrg., 66 (2006), pp. 250-271.

[32] A. LogG, K.-A. Mardal, G. N. Wells, Automated Solution of Differential Equations by the Finite Element Method, Springer, Heidelberg, 2012.

[33] A. Logg And G. N. Wells, Dolfin: Automated finite element computing, ACM Trans. Math. Softw., 37 (2010), 20.

[34] J. Mandel, C. R. Dohrmann, and R. Tezaur, An algebraic theory for primal and dual substructuring methods by constraints, Appl. Numer. Math., 54 (2005), pp. 167-193.

[35] J. MANDEl AND B. SousedíK, Adaptive selection of face coarse degrees of freedom in the BDDC and the FETI-DP iterative substructuring methods, Comput. Methods Appl. Mech. Engrg., 196 (2007), pp. 1389-1399.

[36] J. Mandel, B. Sousedík, and C. R. Dohrmann, Multispace and multilevel BDDC, Computing, 83 (2008), pp. 55-85.

[37] J. Mandel, B. Sousedík, And J. Šístek, Adaptive BDDC in three dimensions, Math. Comput. Simulation, 82 (2012), pp. 1812-1831.

[38] T. P. Mathew, Domain Decomposition and Iterative Refinement Methods for Mixed Finite Element Discretisations of Elliptic Problems, Ph.D. thesis, New York University, Courant Institute of Mathematical Sciences, New York, 1989.

[39] T. P. MATHEW, Schwarz alternating and iterative refinement methods for mixed formulations of elliptic problems. I. Algorithms and numerical results, Numer. Math., 65 (1993), pp. 445468.

[40] T. P. MATHEW, Schwarz alternating and iterative refinement methods for mixed formulations of elliptic problems. II. Convergence theory, Numer. Math., 65 (1993), pp. 469-492.

[41] D.-S. OH, O. B. Widlund, S. Zampini, And C. Dohrmann, BDDC algorithms with deluxe scaling and adaptive selection of primal constraints for Raviart-Thomas vector fields, Math. Comp., to appear, https://doi.org/10.1090/mcom/3254.

[42] L. F. PaVarino And O. B. Widlund, Balancing Neumann-Neumann methods for incompressible Stokes equations, Comm. Pure Appl. Math., 55 (2002), pp. 302-335.

[43] L. F. Pavarino, O. B. Widlund, And S. Zampini, BDDC preconditioners for spectral element discretizations of almost incompressible elasticity in three dimensions, SIAM J. Sci. Comput., 32 (2010), pp. 3604-3626.

Copyright (c) by SIAM. Unauthorized reproduction of this article is prohibited. 
[44] C. Pechstein and C. R. Dohrmann, Modern Domain Decomposition Solvers: BDDC, Deluxe Scaling, and An Algebraic Approach, https://people.ricam.oeaw.ac.at/c.pechstein/ pechstein-bddc2013.pdf (2013).

[45] C. Pechstein and C. R. Dohrmann, A Unified Framework for Adaptive BDDC, http://www. ricam.oeaw.ac.at/files/reports/16/rep16-20.pdf (2016).

[46] J. Š́́steK, J. BŘEzINA, AND B. SOUSEDÍk, BDDC for mixed-hybrid formulation of flow in porous media with combined mesh dimensions, Numer. Linear Algebra Appl., 22 (2015), pp. 903-929.

[47] B. Sousedík, Nested BDDC for a saddle-point problem, Numer. Math., 125 (2013), pp. 761783.

[48] B. Sousedík, J. Š́́stek, And J. Mandel, Adaptive-multilevel BDDC and its parallel implementation, Computing, 95 (2013), pp. 1087-1119.

[49] SPE Comparative Solution Projects, http://www.spe.org/web/csp/datasets/set02.htm.

[50] N. Spillane, V. Dolean, P. Hauret, F. Nataf, C. Pechstein, and R. Scheichl, Abstract robust coarse spaces for systems of PDEs via generalized eigenproblems in the overlaps, Numer. Math., 126 (2013), pp. 741-770.

[51] A. Toselli And O. B. Widlund, Domain decomposition methods-algorithms and theory, Springer Ser. Comput. Math. 34, Springer, Berlin, 2005.

[52] X. TU, A BDDC algorithm for a mixed formulation of flow in porous media, Electron. Trans. Numer. Anal., 20 (2005), pp. 164-179.

[53] X. Tu, BDDC Domain Decomposition Algorithms: Methods with Three Levels and for Flow in Porous Media. Ph.D. thesis, New York University, Courant Institute of Mathematical Sciences, New York, 2006.

[54] X. TU, A BDDC algorithm for flow in porous media with a hybrid finite element discretization, Electron. Trans. Numer. Anal., 26 (2007), pp. 146-160.

[55] X. TU, Three-level BDDC in three dimensions, SIAM J. Sci. Comput., 29 (2007), pp. 17591780.

[56] X. TU, Three-level BDDC in two dimensions, Internat. J. Numer. Methods Engrg., 69 (2007), pp. 33-59.

[57] X. TU, A three-level BDDC algorithm for a saddle point problem, Numer. Math., 119 (2011), pp. 189-217.

[58] X. TU AND J. LI, A unified dual-primal finite element tearing and interconnecting approach for incompressible Stokes equations, Internat. J, Numer. Methods Engrg., 94 (2013), pp. 128149.

[59] X. Tu AND J. LI, A FETI-DP type domain decomposition algorithm for three-dimensional incompressible Stokes equations, SIAM J. Numer. Anal., 53 (2015), pp. 720-742.

[60] M. F. Wheeler, G. Xue, And I. Yotov, A multiscale mortar multipoint flux mixed finite element method, ESAIM Math. Model. Numer. Anal., 46 (2012), pp. 759-796.

[61] O. B. Widlund and C. R. Dohrmann, BDDC deluxe domain decomposition, in Domain Decomposition Methods in Science and Engineering XXII, Lecture Notes Comput. Sci. Eng. 104, Springer, Cham, Switzerland, 2016, pp. 93-103.

[62] B. I. Wohlmuth, A. Toselli, and O. B. Widlund, An iterative substructuring method for Raviart-Thomas vector fields in three dimensions, SIAM J. Numer. Anal., 37 (2000), pp. 1657-1676.

[63] S. ZAMPINI, PCBDDC: A class of robust dual-primal methods in PETSc, SIAM J. Sci. Comput., 38 (2016), pp. S282-S306.

[64] S. ZAMPINI, Adaptive BDDC deluxe methods for H(curl), in Domain Decomposition Methods in Science and Engineering XXIII, C.-O. Lee, X.-C. Cai, D. E. Keyes, H. H. Kim, A. Klawonn, E.-J. Park, and O. B. Widlund, ed., Springer, Cham, Switerland, 2017, pp. 285-292.

[65] S. ZAMPINI AND D. E. KeYES, On the robustness and prospects of adaptive BDDC methods for finite element discretizations of elliptic PDEs with high-contrast coefficients, In Proceedings of the Platform for Advanced Scientific Computing Conference, PASC '16, ACM, New York, 2016, 6.

Copyright $@$ by SIAM. Unauthorized reproduction of this article is prohibited. 